\title{
One-step Synthesis, Biodegradation and Biocompatibility of Polyesters Based on the Metabolic Synthon, Dihydroxyacetone
}
Julius N. Korley ${ }^{1}$, Sara Yazdi ${ }^{1}$, Kevin McHugh ${ }^{4}$, James Kirk $^{4}$, James Anderson $^{3}$, David Putnam ${ }^{1,2,}$

\footnotetext{
${ }^{1}$ Meinig School of Biomedical Engineering and the ${ }^{2}$ Robert Frederick Smith School of Chemical and Biomolecular Engineering, Cornell University, Ithaca NY, 14853.

${ }^{3}$ Department of Pathology and ${ }^{4}$ Department of Biomedical Engineering Case Western Reserve University, Cleveland, $\mathrm{OH} 44106$
}

*To whom correspondence should be addressed: 147 Weill Hall, Cornell University, Ithaca, NY. tel.607-255-4352, fax 607-255-7330, dap43@,cornell.edu 


\section{ABSTRACT}

The one-step synthesis of a polyester family containing dihydroxyacetone is described along with a quantitative analysis of in vitro/in vivo degradation kinetics and initial biocompatibility. Polyesters were synthesized by combining dihydroxyacetone, which is a diol found in the eukaryotic glucose metabolic pathway, with even-carbon aliphatic diacids (adipic, suberic, sebacic) represented in the long-chain alpha carboxylic acid metabolic pathway, by Schötten-Baumann acylation. We show that by using a crystalline monomeric form of dihydroxyacetone, well-defined polyesters can be formed in one step without protection and deprotection strategies. Both diacid length and polyester molecular weight were varied to influence polymer physical and thermal properties. Polyesters were generated with numberaveraged $\left(\mathrm{M}_{\mathrm{n}}\right)$ molecular weights ranging from 2,200-11,500. Polydispersities were consistent with step-growth polymerization and ranged from 2 to 2.6. The melting $\left(\mathrm{T}_{\mathrm{m}}\right)$ and recrystallization $\left(\mathrm{T}_{\mathrm{c}}\right)$ temperatures were impacted in an unpredictable manner. Thermal transitions for the polyesters were highest for the adipic acid followed by suberic acid and sebacic acid, respectively. It was shown that the thermal response of the DHA-based polyesters was influenced by both the diacid length and molecular weight. In vitro degradation studies revealed first-order weight loss kinetics, the molecular weight loss followed first order kinetics with $25 \%$ to $40 \%$ of the original mass remaining after 8 weeks. In vivo testing over 16 weeks highlighted that mass loss ranged from $\sim 70 \%$ to $\sim 6 \%$ depending upon initial molecular weight and diacid length. Histological analysis revealed rapid resolution of both acute and chronic inflammatory responses, normal foreign body responses were observed and no inflammation was present after week 4. This one-step synthesis proved robust with unique copolymers warranting 
further study as potential biomaterials.

Keywords: biodegradation, biocompatibility, kinetics, polyester, dihydroxyacetone, metabolic synthons, biomaterial 


\section{INTRODUCTION}

Dihydroxyacetone-based polymers are materials with potential applications in biomedical devices, tissue engineering matrices and sustainable plastics [1]. To date, polycarbonates and polycarbonate-esters containing dihydroxyacetone (DHA) are reported in the literature [2-7]. The potential medical utility of DHA-based polymers include the prevention of postoperative seroma, the maintenance of hemostasis following organ ligation and the controlled release of medicines

[8-11]. One challenge associated with the synthesis of DHA-based polymers is the intrinsic reactivity and instability of the C2 carbonyl group. While reactivity of DHA's C2 ketone is advantageous when the formation of brown Amadori rearrangement products is desired, like for sunless tanning agents [12-14], its reactivity can be problematic when making polymeric materials. For this reason, the DHA-based polymers reported to date are synthesized from DHA wherein the $\mathrm{C} 2$ ketone is reversibly protected with an acetal, which prevents both ketone reactivity as well as formation of the DHA dimer [2-4,9].

Interestingly, however, even though DHA is a diol, polyesters containing DHA have yet to be reported. Herein, we report a single step synthesis for a class of DHA-containing polyesters (Schötten-Baumann acylation, Scheme 1), quantify and contrast their degradation kinetics (in vitro and in vivo) and characterize their biocompatibility following subcutaneous implantation. The polyesters are composed of DHA and even-carbon diacids. The synthetic route is unique for DHA-based materials since protection/deprotection of the reactive C2 carbonyl is unnecessary, and is made possible through careful crystallization to form the monomeric form of DHA. From a product development perspective, synthesis of a foundational material in one step significantly 
lowers cost and increases potential economic viability. Both the length of the alkane diacid and polymer molecular weights were altered to quantify their influence on the polyester physical characteristics, as well as kinetics of degradation, and all reported polymers showed favorable biocompatibility over 16 weeks, suggesting the potential translatability of the materials as implantable systems.

\section{MATERIALS AND METHODS}

\section{Materials}

Methanol (HPLC grade) and deuterated chloroform (99.8\%, Norell) were purchased from Fisher Scientific. Dihydroxyacetone dimer (DHA, $\geq 95 \%$ purity), adipoyl dichloride $(99.0 \%$ purity), suberoyl dichloride (97\% purity) and sebacoyl dichloride ( $99.0 \%$ purity), pyridine (anhydrous, 99.8\% purity) and chloroform (anhydrous, 99\% purity) were purchased from SigmaAldrich. DHA was further purified by one recrystallization in 2-propanol (Certified A.C.S. grade). Both the pyridine and chloroform were packaged in Sure/Seal ${ }^{\mathrm{TM}}$ containers. All other chemicals were used as received without further purification.

\section{Equipment}

${ }^{1} \mathrm{H}$ and ${ }^{13} \mathrm{C}$ NMR spectra were recorded on a Varian Inova $600 \mathrm{MHz}$ NMR $\left({ }^{13} \mathrm{C}\right.$ NMR, 150.8 MHz) spectrometer in deuterated chloroform. FTIR spectra were recorded on a Bio-Rad FTS 3000MX spectrometer in $\mathrm{KBr}$ pellets (99\% potassium bromide and 1\% polyester). Gel permeation chromatography (GPC) was carried out using a Waters GPC system at $40{ }^{\circ} \mathrm{C}$ with three columns in series, Waters Styragel HR1, HR3 and HR5 columns, and a chloroform mobile phase $(1 \mathrm{~mL} / \mathrm{min})$. Polystyrene standards, from Sigma, were used for relative calibration with RI 
(Waters 2414) detection. Melting point and glass transition measurements were performed on a TA Instruments DSC Q2000 at a heating/cooling rate of $10^{\circ} \mathrm{C}$ per minute and nitrogen flow rate of $50 \mathrm{~mL} / \mathrm{min}$. Calibration was performed using a sapphire standard and adjusted if necessary. A baseline correction was performed using an empty pan reference. TGA experiments were conducted on a TA Instruments TGA Q500 with a heating rate of $10^{\circ} \mathrm{C}$ per minute and nitrogen flow rate of $50 \mathrm{~mL} / \mathrm{min}$. Temperature calibration was performed using high purity calcium oxalate as a reference.

\section{Nomenclature}

The assigned chemical names are based on guidelines in the most recent ACS Division of Polymer Chemistry Macromolecular Nomenclature Note (Note 10) for polyesters. Common names are assigned for identification ease for the reader. Specifically, the common name for poly(1,3-2-oxo-propylene 1,6-hexanedicarboxylate) is poly(DHA-co-adipic acid), the common name for poly(1,3-2-oxo-propylene 1,6-octanedicarboxylate) is poly(DHA-co-suberic acid), and the common name for poly(1,3-2-oxo-propylene 1,6-decanedicarboxylate) is poly(DHA-cosebacic acid). Table 1 depicts polymer nomenclature that is used throughout the manuscript.

\section{General synthetic method}

All polymerizations were conducted using the same synthetic protocol, which is described below for poly(DHA-co-adipic acid). DHA recrystallized from 2-propanol (3.5 g, $0.0388 \mathrm{~mol})$ and chloroform $(150 \mathrm{~mL})$ were combined on a water-ice bath under $\mathrm{N}_{2}$ with stirring in an Airfree ${ }^{\mathbb{R}}$ $500 \mathrm{~mL}$ single neck reaction flask, fitted with a $2 \mathrm{~mm}$ glass stopcock. Adipoyl dichloride (5.67 $\mathrm{mL}, 0.0388 \mathrm{~mol}$ ) was slowly added using a glass syringe and stainless steel needle through a rubber septum at a rate of $1 \mathrm{~mL}$ per minute, immediately followed by pyridine $(7.5 \mathrm{~mL})$ at the 
same rate. After addition of all reagents, the reaction was removed from the water-ice bath and the rate of $\mathrm{N}_{2}$ was reduced. The reaction was vented and allowed to react for predetermined times (monomer composition-dependent) under a blanket of $\mathrm{N}_{2}$. At desired time points, the reaction was transferred to a separatory funnel, washed $4 \mathrm{X}$ with Nanopure, RO water (18.2 $\Omega$, $150 \mathrm{~mL}$ ), the organic phase isolated and reduced by rotoevaporation to $\sim 10 \mathrm{~mL}$. The polymer was precipitated into excess stirring methanol $(500 \mathrm{~mL})$, and the resulting white product was isolated by filtration and dried in vacuo. Each polymer received multiple washes and methanol precipitations until the NMR was free of residual reagents. Poly(DHA-co-adipic acid) yield: 1.7 $\mathrm{g}$ of $\mathrm{M}_{\mathrm{w}}=4,900(17 \%) .{ }^{1} \mathrm{H}$ NMR $\left(\mathrm{CDCl}_{3}\right) \delta(\mathrm{ppm}): 4.76(\mathrm{~s} ; 4 \mathrm{H}), 2.46(\mathrm{t} ; 4 \mathrm{H}), 1.73(\mathrm{q} ; 4 \mathrm{H}) .{ }^{13} \mathrm{C}$ NMR $\left(\mathrm{CDCl}_{3}\right) \delta(\mathrm{ppm}): 198.0,172.5,66.3,33.4,24.2$. Poly(DHA-co-suberic acid) yield: $4.2 \mathrm{~g}$ and $5.3 \mathrm{~g}$ of $\mathrm{M}_{\mathrm{w}}=9,800$ and $\mathrm{M}_{\mathrm{w}}=16,500\left(47 \%\right.$ and $60 \%$, respectively). ${ }^{1} \mathrm{H}$ NMR $\left(\mathrm{CDCl}_{3}\right) \delta$ (ppm): 4.76 (s; 4H), 2.43 (t; 4H), 1.66 (qn; 4H), 1.37 (qn; 4H). ${ }^{13} \mathrm{C}$ NMR $\left(\mathrm{CDCl}_{3}\right) \delta$ (ppm): 197.5, 172.2, 65.6, 28.0, 24.0. Poly(DHA-co-sebacic acid) yield: : $8.4 \mathrm{~g}$ and $8.2 \mathrm{~g}$ of $\mathrm{M}_{\mathrm{w}}=18,700$ ) and $\mathrm{M}_{\mathrm{w}}=29,600$ (84 and 82\%, respectively). ${ }^{1} \mathrm{H}$ NMR $\left(\mathrm{CDCl}_{3}\right) \delta(\mathrm{ppm}): 4.75(\mathrm{~s} ; 4 \mathrm{H}), 2.42(\mathrm{t} ; 4 \mathrm{H})$, 1.65 (qn; 4H), 1.32 (qn; 8H). ${ }^{13} \mathrm{C} \mathrm{NMR}\left(\mathrm{CDCl}_{3}\right) \delta(\mathrm{ppm}): 198,172.7,66.0,33.5,28.8,24.6$.

\section{Reaction Kinetics}

The same synthetic methods described above were used for each polymer, but the total reaction was conducted at $1 / 10^{\text {th }}$ scale (i.e. starting DHA was $0.350 \mathrm{~g}$ instead of $3.5 \mathrm{~g}$ ) in an Airfree ${ }^{\circledR} 100 \mathrm{~mL}$ single neck reaction flask fitted with a $2 \mathrm{~mm}$ glass stopcock. Recrystallized DHA $(0.350 \mathrm{~g}, 0.0039 \mathrm{~mol})$ and chloroform $(15 \mathrm{~mL})$ were combined on a water-ice bath under $\mathrm{N}_{2}$ with stirring. Adipoyl, suberoyl or sebacoyl dichloride $(0.567 \mathrm{~mL}, 0.596 \mathrm{~mL}$ or $0.830 \mathrm{~mL}$ respectively, $0.0039 \mathrm{~mol}$ ) was slowly added using a glass syringe at a rate of $0.1 \mathrm{~mL}$ per minute followed by pyridine $(0.75 \mathrm{~mL})$ at the same rate. After addition of all reagents, the reaction was 
removed from the water-ice bath and the $\mathrm{N}_{2}$ was slowed. The reaction was vented and allowed to react for predetermined times under a blanket of $\mathrm{N}_{2}$. At each desired time point, the reaction was transferred to a separatory funnel, washed $4 \mathrm{X}$ with $15 \mathrm{~mL}$ Nanopure, RO water (18.2 $\Omega$ ), the organic phase isolated and reduced by rotoevaporation to $\sim 1 \mathrm{~mL}$. The polymer was precipitated into excess stirring methanol $(100 \mathrm{~mL})$, isolated by filtration and dried in vacuo. The reaction times were 1, 3, 22, 37, 67, 127, 247 and 487 minute(s). Each reaction was conducted in duplicate and the data points shown are an average of the two independent experiments.

\section{Sample Preparation and Sterilization}

Pellets were fabricated by melt extrusion for both the in vitro and in vivo studies. Melt extrusion was used since films formed by traditional solvent casting yielded brittle films. Pellets were fabricated by melting polymers above their respective $T_{m}$, followed by aspiration of the melt by syringe suction into a $2 \mathrm{~mm}$ ID high-density polypropylene tube $(7.0 \mathrm{~cm})$. Polymer was allowed to cool for a few seconds at room temperature, then released from the polypropylene tube though insertion of plunger. The released polymer was cut into $5.0 \mathrm{~mm}$ lengths and weighed. Samples were sterilized by immersion in $100 \%$ ethanol for 5 minutes. For the in vitro studies, all instruments required for the experiment were autoclaved and thoroughly cleaned with $100 \%$ ethanol to maintain sterility. For the in vivo studies, all surgical instruments were sterilized prior to surgery by autoclave and between use in each animal by a glass bead sterilizer (Germinator 500, Cellpoint Scientific, Rockville, MD).

Phosphate buffered saline (10X PBS; Gibco Laboratories, Grand Island, NY, USA) without $\mathrm{Ca}^{2+}$ and $\mathrm{Mg}^{2+}$ was used to make both the $\mathrm{pH} 3.0$ and 5.0 solutions. For each solution, the 10X stock was diluted to $1 \mathrm{X}$ using Nanopure, RO water $(18.2 \Omega)$. The $\mathrm{pH}$ of each solution 
was then adjusted appropriately using concentrated hydrochloric acid $(\mathrm{HCl}$; Thermo Fisher Scientific Inc., Fairlawn, NJ) and measured using a pH meter (Mettler Toledo, Columbus $\mathrm{OH}$ ). Solutions were sterile filtered before use. Phosphate buffered saline, 1X, (PBS; Gibco Laboratories, Grand Island, NY, USA) without $\mathrm{Ca}^{2+}$ and $\mathrm{Mg}^{2+}, \mathrm{pH} 7.4$ was used as purchased.

\section{In Vitro Degradation and Analysis}

Polyester pellets were placed inside the wells of non-treated 24-well tissue culture plates (TCPS; Becton Dickinson, Sparks, MD, USA). Buffer $(2 \mathrm{~mL})$ was added to each well to completely submerge each pellet. Blunt forceps was used to place floating pellets at the bottom of the plates. Upon insuring complete submersion of each pellet, each plate was maintained in a humidified incubator at $37^{\circ} \mathrm{C}$. Triplicate samples were used for each time point and each polyester type. Each $\mathrm{pH}$ condition was conducted in separate tissue culture plates. Evaporation was controlled for by weekly inspection, removal of buffer (frozen and retained at $-20{ }^{\circ} \mathrm{C}$ ), and replacement with fresh pre-warmed buffer. To insure that the $\mathrm{pH}$ remained constant for each sample, a control plate under each condition was maintained in parallel and evaluated for $\mathrm{pH}$ change throughout the course of the experiment. Experimental pellet samples were removed at 1, 2, 4, and 8 weeks. Each sample was rinsed with ethanol (100\%) and dried in a vacuum oven at $45^{\circ} \mathrm{C}$ for at least 48 hours. The samples were then weighed and subjected to molecular weight analysis by GPC.

\section{In Vivo Subcutaneous Implantation}

Polyesters pellets were implanted subcutaneously, four per animal, into 12 week-old female Sprague Dawley Rats (Charles Rivers Laboratories, North Wilmington, MA). The implants were then removed at 1,2, 4, 6, 8, 12 and 16 weeks post surgery and analyzed by 
histological grading, molecular weight analysis, and gravimetric mass loss. All surgeries were performed in the Animal Resource Center (ARC) at Case Western Reserve University under protocols approved by the Institutional Animal Care and Use Committee (IACUC). Proper care for laboratory animals was provided under NIH guidelines both during and after the surgeries. Rats were fed ad libitum and post-operative care was provided by ARC veterinary technicians.

The rats were anesthetized using Aerrane ${ }^{\circledR}$ isoflurane anesthetic liquid for inhalation (Baxter, Deerfield, IL) in a bell jar and received a continuous dosage throughout the surgery. The back of the rats were shaved using an electric razor and remaining adherent hair was removed using water. The site was then cleaned with Betadine ${ }^{\circledR}$ (Purdue Frederick, Stamford, CT) to maintain an aseptic surgical site. The subcutaneous implantation of biomaterials has been previously described in detail [15-18]. A 1.0 to $1.5 \mathrm{~cm}$ incision was made along the posterior midline of the back. Marcaine ${ }^{\circledR}$ topical anesthetic (Abbott Laboratories, North Chicago, IL) was then locally applied to the incision to help minimize potential post-operative pain. Four subcutaneous pockets were cleared on either side of the midline below the panniculus carnosus in the connective tissue fascia using blunt dissection with rounded-tip scissors. One sample was placed into each pocket ensuring sufficient distance from the midline and between pockets to minimize material migration. The wound site was closed using $9 \mathrm{~mm}$ surgical wound clips and cleaned using a second application of Betadine ${ }^{\circledR}$. Each rat was then returned to its cage and observed until it recovered from anesthesia. This procedure was repeated for a total of 56 animals to achieve $\mathrm{n}=8$ ( $\mathrm{n}=4$ for histological analysis and $\mathrm{n}=4$ for material degradation studies). All four materials were implanted into each animal, at different anatomical positions, to minimize animal-to-animal host response variations.

\section{In Vivo Subcutaneous Explantation}


Pellets were explanted at $1,2,4,6,8,12$, and 16 weeks in vivo. At each time point 8 animals were euthanized by carbon dioxide inhalation. Pellets from four animals were excised with the surrounding tissue intact $(5 \mathrm{~cm} \times 5 \mathrm{~cm})$ to preserve the integrity of the fibrous capsule and to include all tissue surrounding the polymer. These samples were fixed for 48 hours in $10 \%$ buffered formalin phosphate solution (Fisher Scientific, Fairlawn, NJ) for histological analysis. The remaining polymer pellets were explanted from the four remaining animals and freed completely of adherent tissue for mass analyses (molecular weight and mass loss).

The fixed tissue was cut into $1-2 \mathrm{~mm}$ wide sections at the tissue-polymer interface to obtain a cross-sectional view of the fibrous capsule and surrounding cells. The total number of sections ranged between 3 and 4 . The sections were then imbedded in paraffin blocks that were subsequently cut into $4 \mu \mathrm{m}$ thick sections and mounted onto slides.

\section{Histological Analysis}

Histological analysis was performed on all sections using light microscopy. Each slide was stained with either Hematoxylin or Eosin (H\&E) or Masson's Trichrome (University Hospitals of Cleveland, Histopathology Laboratory; Cleveland, OH). The slides were photographed using light microscopy (Olympus BH-2) and Nikon-ACT Software Imaging System $^{\circledR}$ (Nikon DXM1200 Digital Camera; Product No. 12260). The extent and intensity of the inflammatory response was determined on $\mathrm{H} \& \mathrm{E}$ stained tissue sections and characterization of the collagen content of the capsule by using Masson's Trichrome stain. The scoring system used assigns a number, from 0 to +4 , to each section at each time point, where $0=$ none, $+1=$ minimal, $+2=$ mild, $+3=$ moderate, and $+4=$ extensive. Each section was scored on all phases of the inflammatory and wound healing response (acute inflammation, chronic inflammation, 
granulation tissue, and foreign body reaction), and fibrous capsule structure (maturity). The stages of the wound healing continuum or foreign body reaction following biomaterial implantation have been well characterized [19].

\section{Statistical Analysis}

Statistical analysis for the animal work was carried out using two-way ANOVA for repeated measures, followed by Bonferroni $t$ post hoc testing on Prism 5.0 for Macintosh (Graphpad, La Jolla, CA). A p-value equal or lower than 0.05 was considered statistically significant and is denoted by $(* *)$.

\section{Determination of the Degradation Rate Constant, "k"}

To determine the degradation rate constant (k), ln $\left(\mathrm{M}_{\mathrm{w}^{\prime} t} / \mathrm{M}_{\mathrm{w}, 0}\right)$ versus time (wk), was plotted where $M_{w^{\prime} t}$ is the observed $M_{w}$ at time (t) and $M_{w, 0}$ is the $M_{w}$ of the control (non-treated) pellet. A linear trend line was generated with the equation for the line in the slope-intercept form, or $y=m x+b$, where $m$ equals the slope and $b$ equals the $y$-intercept. The rate constant, " $k$ ", equals the negative slope. 


\section{RESULTS}

\section{Polyester Synthesis and Polymerization Kinetics}

To determine molecular weight as a function of reaction time, and to determine the reaction time required for each polymer to reach the maximum molecular weight, a kinetic analysis of the reaction was conducted (Figure 1). Time zero was designated as the time when pyridine addition was initiated. As the length of the diacid increased, so did the rate of the initial increase in $\mathrm{M}_{\mathrm{W}}$ of the resulting polymer. The increase in molecular weight reached a plateau near the $2 \mathrm{hr}$ time point for each synthesis. The $\mathrm{M}_{\mathrm{w}}$ peaked at $\sim 2.1 \times 10^{4}$ for the two larger diacids but showed a maximum of $1.0 \times 10^{4}$ for the adipic acid-containing copolymer. Additionally, the polydispersity indices (PDI) for each polymer was $\sim 2.0$, which is common for step growth polymerizations (Figure 2) [20]. Based on the reaction kinetics, approximate times were chosen to generate molecular weights at or near the maximum achieved during the kinetics study. Furthermore, we aimed to generate lower molecular weights of $\sim 1.0 \times 10^{4}$ to allow an investigation into the effect of $\mathrm{M}_{\mathrm{w}}$ on the polymers' thermal and degradation characteristics; however, this was not possible for the adipic acid copolymer since that was the maximum molecular weight achievable. The polymerization kinetics data generated using smaller batch sizes did not scale linearly with the large batch sizes; therefore, reaction times for larger batches were adjusted to achieve the desired $\mathrm{M}_{\mathrm{w}}$ (Table 1). For the large batch reactions, the maximum $\mathrm{M}_{\mathrm{w}}$ for the sebacic acid copolymer reached $3.0 \times 10^{4}$. The $\mathrm{M}_{\mathrm{w}}$ of the suberic acid copolymer remained unchanged while the adipic acid copolymer maximum $\mathrm{M}_{\mathrm{w}}$ decreased to about $5.0 \mathrm{x}$ $10^{3}$. 


\section{Chemical and Thermal Characterization}

Figure 3 shows annotated ${ }^{1} \mathrm{H}$ NMR and ${ }^{13} \mathrm{C}$ NMR spectra for the copolymers. These spectra of poly(DHA-co-sebacic acid) (29.6k) are representative of the spectra for all synthesized copolymers listed in Table 1. For both the proton and carbon spectra, the peaks denoted by letters are assigned the appropriate signals in the repeating units of the polymers. Both the ${ }^{1} \mathrm{H}$ and ${ }^{13} \mathrm{C}$ NMR spectra for each copolymer confirm the proposed molecular structure of the polymers. Because of the symmetry of the polymer, many of the peaks were chemically equivalent and thus show up in the spectrum as a single peak, and this was confirmed by integration.

Figure 4 shows a representative infrared spectrum for poly(DHA-co-sebacic acid) (29.6k). Five functional groups, both a symmetrical and asymmetrical carbon-oxygen stretch (CO) within the "fingerprint" region, a carbonyl peak $(\mathrm{C}=\mathrm{O})$, an alkane peak $(\mathrm{C}-\mathrm{H})$, and an alcohol stretch $(\mathrm{O}-\mathrm{H})$, were all observed with strong absorbance at $\approx 1150 \mathrm{~cm}^{-1}, 1728 \mathrm{~cm}^{-1}, 2900 \mathrm{~cm}^{-1}$, and $3400 \mathrm{~cm}^{-1}$ respectively. Collectively, the NMR and FTIR spectra support the proposed structure of the polyesters.

Thermally-induced changes in the copolymers were characterized by differential scanning calorimetry (DSC) and thermal gravimetric analysis (TGA). DHA is a solid at room temperature with a melting temperature $\left(\mathrm{T}_{\mathrm{m}}\right)$ of $80^{\circ} \mathrm{C}$. All of the copolymers have a $\mathrm{T}_{\mathrm{m}}\left(2^{\text {nd }}\right.$ heating) ranging from $101^{\circ} \mathrm{C}$ to $119^{\circ} \mathrm{C}$ as determined by DSC (Table 2). Upon cooling, the copolymers exhibit apparent crystallization temperatures $\left(\mathrm{T}_{\mathrm{c}}\right)$ ranging from $71{ }^{\circ} \mathrm{C}$ to $93^{\circ} \mathrm{C}$ depending upon molecular weight and monomer composition. Similar crystallization behavior has been observed in other polyesters [21,22]. No glass transition temperatures $\left(T_{g}\right)$ were observed for all copolymers using DSC. Using TGA, the temperature at which $0.5 \%$ weight loss was detected ranged from $139^{\circ} \mathrm{C}$ to $191^{\circ} \mathrm{C}$ (Table 3). 


\section{In Vitro Degradation}

Polyester pellets were incubated for $1,2,4$, and 8 weeks under three different $\mathrm{pH}$ conditions ( $\mathrm{pH}$ 3.0, 5.0 and 7.4) and subjected to molecular weight analyses by GPC. The 3 different $\mathrm{pH}$ conditions were chosen in an effort to help confirm the mechanism of degradation. As noted in Anderson et al in their thorough review of PLA and PLGA biodegradation and biocompatibility, foreign body giant cells (FBGC) and macrophages can produce acid amongst others agents to aid in biodegradation process [23]. Figure 5, which shows the percent of molecular weight loss as a function of time and $\mathrm{pH}$ for poly(DHA-co-sebacic acid) (29.6k), is representative of the degradation characteristics for all copolymers, and shows the rapid reduction in $\mathrm{M}_{\mathrm{w}}$ over the first few weeks followed by a period of slower degradation thereafter for each $\mathrm{pH}$ condition. Over the eight-week incubation period, the white to off-white pellets changed in color to a yellowish-brown, which is consistent with the formation of free DHA. The integrity of each pellet was not compromised over the course of the experiment as there was no breaking or crumbling of the pellet nor were the pellets frail during manipulation. No more than $6 \%$ mass loss was observed for any of the polymers. The degradation rate constants were calculated for the initial degradation phase $\left(\mathrm{k}_{1}\right)$ and the slower long-term degradation phase $\left(\mathrm{k}_{2}\right)$, when applicable, assuming first order kinetics. The correlative half-lives $\left(t_{1 / 2}\right.$ and $\left.t_{2(1 / 2)}\right)$ were also calculated and tabulated in Table 4. The degradation kinetics were unique for each polymer with respect to $\mathrm{pH}$, and there was no general correlation between molecular weight, polymer type and degradation profile.

\section{In Vivo Degradation}

Polymer pellets were sterilized by ethanol immersion and surgically implanted subcutaneously into female, 12-week old, Sprague-Dawley rats. At weeks 1, 2, 4, 6, 8, 12 and 16, the pellets were explanted for analysis. Each polymer, implanted as white or off-white 
pellets, changed color during in vivo incubation, and transitioned from yellow to dark brown, as the implantation time increased. Unlike the in vitro degradation studies, the physical integrity of the pellets changed from brittle to liquid-filled to completely degraded (e.g. disappeared) as the implantation time increased. Fragmented pellets were common. Following explantation, all collected polymers were weighed to determine mass loss, then analyzed by GPC to assess the molecular weight and biodegradation patter. A representative degradation profile is shown in Figure 6 for poly(DHA-co-suberic acid $(9.8 \mathrm{~K})$.

For poly(DHA-co-suberic acid) (9.8k) and poly(DHA-co-suberic acid) (16.5k), loss of pellet integrity began at week 8 . At week 16,3 of the 4 implanted pellets of poly(DHA-cosuberic acid) (9.8k) and 1 of 4 pellets of poly(DHA-co-suberic acid) (16.5k) pellets were fully resorbed, with no measurable mass. At week 16 , only $3 \%$ of the pellet mass remained in the single residual sample, but no discernable molecular weight was detectable. For poly(DHA-cosuberic acid) (16.5k), 17\% of the mass remained for the surviving pellets, but again with no meaningful molecular weight.

Poly(DHA-co-sebacic acid) (18.7k) and poly(DHA-co-sebacic acid) (29.6k) were substantially more durable as the integrity of each pellet was uncompromised until week 16. All pellets were able to be recovered at week 16 and were subjected to mass loss and molecular weight analysis. While greater than 70\% mass remained for poly(DHA-co-sebacic acid) (18.7k) and poly(DHA-co-sebacic acid) $(29.6 \mathrm{k})$ at week 16 , the molecular weight profile was indeterminable as only low molecular weight degradation products remained.

\section{In Vivo Biocompatibility}

Sterilized polymer pellets were implanted subcutaneously into female, 12-week old, Sprague-Dawley rats. At 1, 2, 3, 4, 8 and 12 weeks, the pellets were explanted for analysis. 
Similar to the biodegradation study pellets, each polymer, implanted as white or off-white pellets, changed color during in vivo incubation, and transitioned from yellow to dark brown, as the implantation time increased. Additionally, the physical integrity of the pellets changed from brittle to liquid-filled to completely degraded (e.g. disappeared) as the implantation time increased. Fragmented pellets were common. Histology was used to evaluate the host response to the implanted polymers over time. Representative tissue sections at week 1 are shown in Figure 7, with hematoxylin and eosin stain (left) and Masson's trichrome (right).

The wound healing responses of the four polyesters was evaluated at discrete time points: $1,2,4,6,8,12$, and 16 weeks. After 1 week of implantation, no acute inflammation or polymer biodegradation was observed and minimal to mild levels of chronic inflammation, granulation tissue, foreign body reaction and fibrous capsule maturation were observed for any of the polymers tested. After 2 weeks of implantation, foreign body reaction and fibrous capsule development increased to mild and moderate levels, respectively. Chronic inflammation and granulation tissue decreased to minimal by week 4 of implantation. No chronic inflammation or granulation tissue was observed and this trend continued until week 16. Minimal to mild foreign body reaction was present and the fibrous capsule maturation was unchanged from week 2 .

At week 6 , the foreign body reaction had decreased to minimal, showing a trend of steady decrease since week 2 . The fibrous capsule maturation increased slightly to moderate and remained unchanged for the duration of the direct subcutaneous experimentation. There were no significant changes from week 6 to week 8 . Until week 12, there were no significant changes in scoring between any of the polymers tested.

After 12 weeks of implantation, poly(DHA-co-suberic acid) (9.8k) and poly(DHA-cosuberic acid) (16.5k) showed significant increases in foreign body reaction to moderate and mild, 
respectively. Also for the first time, biodegradation was observed for both poly(DHA-co-suberic acid) $(9.8 \mathrm{k})$ and poly(DHA-co-suberic acid) (16.5k) with poly(DHA-co-suberic acid) (9.8k) and poly(DHA-co-suberic acid) (16.5k) reaching mild and minimal, respectively.

At week 16, poly(DHA-co-suberic acid) (9.8k) and poly(DHA-co-suberic acid) (16.5k) showed mild to moderate levels of granulation tissue, foreign body reaction and biodegradation. Poly(DHA-co-sebacic acid) (18.7k) and poly(DHA-co-sebacic acid) (29.6k) continued to show minimal levels of foreign body reaction. Fibrous capsule maturation remained moderate for all polymers.

\section{DISCUSSION}

Because DHA and even-carbon diacids are components in the human metabolome, they have been previously explored as building blocks for implantable polymeric biomaterials $[2,4,8$ $11,24,25]$. However, the synthesis of DHA-containing polymers can be problematic for two reasons. First the $\mathrm{C} 2$ carbonyl of DHA is highly reactive and forms a number of adducts under different conditions [26,27]. Second, DHA exists as both a monomer and as a dimer in solution [28]. To circumvent the aforementioned problems, we considered the work of Bentley and McCrae who successfully made symmetric lipids containing DHA in high yield via a one-step Schötten-Baumann acylation (Scheme 1) [29]. Our reasoning was that if DHA-based symmetric lipids could be made without DHA protection, and if the fidelity of the reaction was high, then the same methodology could be used to synthesize DHA-based polyesters. Therefore, using this approach, DHA dimer was recrystallized in 2-propanol to form monomeric DHA, which was then suspended in anhydrous chloroform in a water-ice bath followed by the dropwise addition of the appropriate diacid chloride. Pyridine was then slowly added dropwise to the reaction to 
capture the resulting hydrochloric acid. The slow addition of pyridine was intentional to prevent its potentially adverse effect on the molecular weight when in excess.

The synthetic approach worked for all three diacids investigated. To better understand how the copolymer molecular weight influenced thermal and degradation behavior, two polymer lengths were targeted for each copolymer. However, after multiple attempts, a meaningful higher molecular weight material was not achievable for the adipic acid-containing copolymer. Therefore, we continued work using one adipic acid-containing copolymer, and two each of the suberic and sebacic acid-containing copolymers.

The thermal characteristics of the polyesters are consistent with the monomer compositions and synthetic route, with broad and sometimes multiple melting points observed. Such thermal characteristics are common for lower molecular weight polymers synthesized by step-wise polymerization where the end group effects and polydispersity can contribute significantly to changes in thermal transition kinetics. The presence of multiple melting peaks indicates a complexity of thermal transitions sometimes seen in polymers that are attributed to melting-recrystalization-remelting steps [30] or to the presence of multiple independent crystalline populations [31]. Generally the longer diacid aliphatic chains elicited higher $T_{d}$. Given no definitive $T_{g}$ was identified and both a $T_{c}$ and $T_{m}$ were observed, the data suggest that these copolymers are crystalline or semi-crystalline materials. The crystalline state of the copolymers is also supported by our substantial efforts to cast continuous films from the polymers using a variety of methods. Minimal success to form high integrity films was achieved, with the best results coming from melt casting under vacuum. While higher molecular weights could lead to films with better integrity, for the current materials even the highest $M_{w}$ copolymers could not form cohesive thin films. 
Table 4 lists copolymer in vitro half-lives and degradation rate constants. Near log-linear degradation was observed for all polymers tested at both $\mathrm{pHs} 3.0$ and 5.0 conditions with best fits associated with pseudo first order kinetics (data not shown). However, at $\mathrm{pH} 7.4$, the results were not log-linear, and furthermore, the data suggests biphasic kinetics governing the molecular weight loss. Based in part on work of Kenley et al, there are a number of mechanistic explanations for the observed degradation pattern (e.g., crystalline/amorphous domain changes, hydrophobic/hydrophilic balance of matrix, etc) [32]. These results are consistent with our previous reports on DHA-polymers wherein the rate of matrix degradation increase with increasing DHA content, with commensurate formation of pores into the matrix that can alter degradation rate [24]. There is also a correlation of these results to the mass loss patterns observed for homopolymers and copolymers of lactic acid and glycolic acid [23,33-35], which are consistent with the general mechanism of polyester bulk erosion and the corresponding formation of diffusionally trapped acids within the matrix leading to acid catalyzed hydrolysis $[35-40]$.

The effects of $\mathrm{pH}$ on the degradation rates for poly(DHA-co-suberic acid) $(9.8 \mathrm{k})$, poly(DHA-co-suberic acid) (16.5k), poly(DHA-co-sebacic acid) (18.7k), and poly(DHA-cosebacic acid) (29.6k) are interesting and highly dependent on the copolymer composition. For poly(DHA-co-suberic acid) (9.8k) and poly(DHA-co-suberic acid) (16.5k), there is increased rate of degradation at the lower $\mathrm{pHs}$ but this is reversed for poly(DHA-co-sebacic acid) (18.7k) and poly(DHA-co-sebacic acid) (29.6k), where decreased rates of degradation were observed at the lower pHs. It is unclear what leads to these interesting results and we are currently working to better understand the underlying degradation mechanism(s) of the DHA-based materials. 
The in vivo degradation studies showed significant differences in the pattern of degradation for molecular weight loss and pellet mass loss. Figure 6, showing the degradation pattern of poly(DHA-co-suberic acid $(9.8 \mathrm{~K})$, is illustrative of the in vivo degradation profiles for each polymer (Figures S1-S3). Copolymers that underwent the same pelleting process, but were not implanted, served as initial state samples. As shown in Figure 6, a 5.0x10 drop in molecular weight was observed in the polymer following week one, which correlates to about $50 \%$ of the molecular weight for poly(DHA-co-suberic acid) $(9.8 \mathrm{k})$ or one half-life. Table 5 lists the in vivo half-lives and degradation rates for all four copolymers. The values listed are through at least two half-lives of in vivo degradation, which is the standard timeframe for biomaterial in vivo degradation studies. Two half-lives is a good a point of reference because linearity becomes compromised from the onset of multiple degradation mechanisms beyond that timeframe. By observing degradation over two half-lives, a predominant mechanism for degradation can be interpreted with a high degree of accuracy $[32,41-44]$. The polymer molecular weight half-life increased with the molecular weight of the polyester, which is consistent with historical polyester degradation behavior. The half-life for this group of polyesters ranged from 2.2 to 4.3 weeks with $\mathrm{k}$ values ranging from 0.330 to $0.162 \mathrm{wk}^{-1}$, and with the degradation rate decreasing as the molecular weight of the polymer increased, [poly(DHA-co-suberic acid) $(9.8 \mathrm{k})]>[$ poly(DHA$c o$-suberic acid) $(16.5 \mathrm{k})]>[$ poly $($ DHA-co-sebacic acid $)(18.7 \mathrm{k})]>[$ poly $(\mathrm{DHA}-c o$-sebacic acid $)$ $(29.6 \mathrm{k})]$.

The mass loss did not correspond with molecular weight loss. For instance, after one halflife, less than $20 \%$ of the mass had been lost for poly(DHA-co-suberic acid) (9.8k) (Figure 6). This trend was consistent for all four implanted polymers. By the end of week 16, poly(DHA-cosuberic acid) (9.8k) and poly(DHA-co-suberic acid) (16.5k) had remaining masses of 3\% and 
$17 \%$, respectively. Both poly(DHA-co-sebacic acid) (18.7k) and poly(DHA-co-sebacic acid) (29.6k) had remaining masses exceeding 70\%. The polymers presented with liquefied interiors starting between weeks two and four, indicative of the acid-catalyzed hydrolysis common to polyesters degradation, first reported by Vert and colleagues [45] where in the entrapped carboxylic acid groups of oligomeric polymer acidify the interior of the copolymer matrix and increases the kinetics of hydrolysis [42].

Subcutaneous implantation in rats was used to evaluate the biocompatibility of the polymers. The implantation procedure causes injury at the implantation site which triggers a wound healing response continuum. The development of a fibrous capsule is indicative of the tissue or cellular host entering the latter stages of the wound healing cascade. Typically, biodegradable polymers requiring extended periods of time before biodegradation have mature fibrous capsule development by week four of implantation. The resolution or absence of acute inflammation after the first week of implantation is indicative that the other responses observed are mainly due to the injury of implantation. As seen earlier in the "Histological analysis" section, the scoring system used assigns a number, from 0 to +4 , to each section at each time point, where $0=$ none,$+1=$ minimal,$+2=$ mild,$+3=$ moderate, and $+4=$ extensive. Each section was scored on all phases of the inflammatory and wound healing response (acute inflammation, chronic inflammation, granulation tissue, and foreign body reaction), and fibrous capsule structure (maturity). Biodegradation/fragmentation was also scored using the same scale.

We analyzed several different outcomes of our polymer treatment and a representative sample is shown in Figure 8. Acute inflammation is resolved by week 1 as indicated by the lack of polymorphonuclear leukocytes in the tissue immediately surrounding the implant. No 
statistical analyses were performed on this outcome as all observed scoring values were " 0 " and thus of no statistical significance.

Chronic inflammation is evidenced by the presence of macrophages and lymphocytes. Chronic inflammation is present at similar levels starting at week one in all of the polymer treatments and is completely resolved by week four (Two-Way ANOVA main effect time $\mathrm{F}_{6,72}$ $=39.52, \mathrm{p}<0.0001)$. No difference between the different polymers is observed on this parameter.

Granulation tissue is another stage of the wound healing cascade indicating the beginning of inflammation resolution, and is evidenced by the presence of angiogenesis and fibroblast infiltration. All of the polymers used in this study induce granulation in the area of implantation (Two-Way ANOVA main effect treatment: $F_{3,72}=21.00, p<0.0001$ ). The presence of granulation changes over time, it is present at weeks one and two (Two-Way ANOVA main effect time: $\mathrm{F}_{6,72}$ $=88.64, \mathrm{p}<0.0001)$ and it is completely resolved by week four. Interestingly there is a recurrence of the granulation at week 12 for two of the polymers used, poly(DHA-co-suberic acid) (9.8k) and poly(DHA-co-suberic acid) (16.5k) (Two-Way ANOVA interaction treatment $\mathrm{x}$ time $\mathrm{F}_{18,72}$ $=17.82, \mathrm{p}<0.0001$, Bonferroni $t 9.8$ or 16.5 vs 18.7 and $29.6 \mathrm{p} \leq 0.001)$. This effect is accompanied by a significant fragmentation of the polymer.

Foreign body reaction is denoted by the presence of macrophages and the appearance of foreign body giant cells (FBGC). This particular outcome is clearly seen at week two and remains for the duration of experimentation. The tissue surrounding the implantation site shows an initial reaction at week two for all the polymers used (Two-Way ANOVA main effect treatment: $\left.F_{3,72}=27.33, p<0.0001\right)$. The foreign body reaction is then maintained for the reminder of the study time (Two-Way ANOVA main effect time: $F_{6,72}=20.44, p<0.0001$ ) for all of the polymers. Interestingly, while both the poly(DHA-co-sebacic acid) (18.7k) and poly(DHA-co- 
sebacic acid) (29.6k) do not induce any further reaction in the tissue surrounding the implantation site, poly(DHA-co-suberic acid) (9.8k) and poly(DHA-co-suberic acid) (16.5k), induce more foreign body reaction at weeks 12 and 16 (Two-Way ANOVA interaction treatment $\mathrm{x}$ time $\left.\mathrm{F}_{18,72}=8.26, \mathrm{p}<0.0001\right)$. This increase in foreign body reaction can be attributed to the increase in surface area of the polymers due to fragmentation. The Bonferroni analysis reveals that poly(DHA-co-suberic acid) (9.8k) vs poly(DHA-co-suberic acid) (16.5k) are different at week 12, poly(DHA-co-suberic acid) (9.8k) vs poly(DHA-co-sebacic acid) (18.7k) are different on week 12 and 16 and poly(DHA-co-suberic acid) (9.8k) vs poly(DHA-co-sebacic acid) (29.6k) are different on week 12 and 16 (all with $\mathrm{p}<0.001$ ).

A mature fibrous capsule is a hallmark of the final stages of the wound healing cascade, and it is clearly identified by using Masson's Trichrome. A fibrous capsule forms around all of the polymers within the first 2 weeks (Two-Way ANOVA main effect treatment: $F_{3,72}=0.61$, n.s.). The maturity of the fibrous capsule holds steadily at moderate levels from weeks four to 16 (Two-Way ANOVA main effect time $\mathrm{F}_{6,72}=26.58, \mathrm{p}<0.0001$ ) for all of the treatments). It is interesting to note that by week 16, poly(DHA-co-suberic acid) $(9.8 \mathrm{k})$ and poly(DHA-co-suberic acid) (16.5k) have been completely degraded and only a collagenous scar remains as previously reported in the literature $[23,46,47]$.

Biodegradation/fragmentation of the polymer pellets has also been observed and measured. It is interesting that biodegradation is observed beginning at week 12 only by the fragmentation of the pellets of poly(DHA-co-suberic acid) (9.8k) and poly(DHA-co-suberic acid) (16.5k). Fragmentation of the pellets made of poly(DHA-co-suberic acid) (9.8k) and poly(DHA-co-suberic acid) (16.5k) reaches its maximum on week 16 (Two-Way ANOVA interaction treatment $\mathrm{x}$ time $\left.\mathrm{F}_{18,72}=18.67, \mathrm{p}<0.0001\right)$ which indicates that different polymers 
behave differently over time. The Bonferroni analysis reveals that poly(DHA-co-suberic acid) (9.8k) vs poly(DHA-co-suberic acid) (16.5k) are different on week 12, poly(DHA-co-suberic acid) (9.8k) vs poly(DHA-co-sebacic acid) (18.7k) are different on week 12 and 16 and poly(DHA-co-suberic acid) (9.8k) vs poly(DHA-co-sebacic acid) (29.6k) are different on week 12 and 16 (all with $\mathrm{p}<0.001$ ). While parallel investigation of FDA-approved polyesters, such as PLGA, we not part of the experiments reported herein (owing to their extensive representation in the literature), these DHA-based polyesters behaved similarly to PLGA as well as other biocompatible and biodegradable polymeric systems known to be safe and effective as potential drug delivery systems $[23,46,47]$. Specifically, the DHA-based polyesters investigated did not exhibit toxicity, did not elicit inflammation and degraded via the well-known mechanism of bulk erosion [23,33-35]. Furthermore, none of the observations within the tissue response continuum used, in part, to determine both biodegradation and biocompatibility were beyond that of other well-studied, FDA-cleared polymers such as PLGA [48-52].

\section{CONCLUSIONS}

Here we report the one-step synthesis and characterization of a new series of polyesters based on metabolic synthons. The polyesters are comprised of the glycolytic intermediate, dihydroxyacetone and even-carbon diacids that are readily metabolized in the human body. The premise of their synthesis was to create potentially useful biomaterials with favorable biocompatibility. A successful one-step method is described with yields reaching $85 \%$. This application of the Schötten-Baumann acylation method provides an easy synthetic route to generate this class of polyesters. The synthesis was performed with relative ease and is also a 
relatively quick reaction. The kinetic studies showed that reasonably high molecular weight polymers could be generated in less than four hours.

Successful synthesis of the target copolymers was confirmed by ${ }^{1} \mathrm{H}$ NMR and ${ }^{13} \mathrm{C}$ NMR and characterized by GPC, TGA and DSC. As the diacid length increases, the yield and $\mathrm{M}_{\mathrm{w}}$ also increase, but diacids shorter than adipic acid were unsuitable monomers for this synthetic route. The polymers each had unique degradation profiles both in vitro and in vivo and were both molecular weight and monomer composition-dependent. The biocompatibility profiles were favorable and in line with other prominently used implantable polyesters such as, PLA and PLGA [23,46,47]. Collectively, these results confirm that this new class of polyesters has favorable biocompatibility and that the degradation products are locally non-toxic. Investigation into the potential clinical application of these polymers is ongoing and they may offer some unique characteristics for implantable devices.

\section{ACKNOWLEDGEMENTS}

This work was supported in part from the Wallace H. Coulter Foundation and the National Science Foundation CAREER Award. This work made use of the Cornell Center for Materials Research Shared Facilities which are supported through the NSF MRSEC program (DMR-1120296). J.N.K would also like to thank the Alfred P. Sloan Foundation for a Research Fellowship. We are grateful to Dr. Stuart Rowan for use of the NMR Facilities and valuable discussions, to Dr. LaShanda Korley for laboratory space and use of the TA Thermal Analysis suite and to Dr. Ippolita Cantuti-Castelvetri for help with the statistical analysis. We thank Drs. David Schiraldi and Thomas H. Mourey for their valuable contributions. 


\section{REFERENCES}

[1] N.G. Ricapito, C. Ghobril, H. Zhang, M.W. Grinstaff, D. Putnam, Synthetic Biomaterials from Metabolically Derived Synthons, Chem. Rev. (2016) acs.chemrev.5b00465. doi:10.1021/acs.chemrev.5b00465.

[2] A.N. Zelikin, P.N. Zawaneh, D. Putnam, A Functionalizable Biomaterial Based on Dihydroxyacetone, an Intermediate of Glucose Metabolism, Biomacromolecules. 7 (2006) 3239-3244.

[3] A.N. Zelikin, D. Putnam, Poly(carbonate-acetal)s from the Dimer Form of Dihydroxyacetone, Macromolecules. 38 (2005) 5532-5537. doi:10.1021/ma050049v.

[4] P.N. Zawaneh, A.M. Doody, A.N. Zelikin, D. Putnam, Diblock Copolymers Based on Dihydroxyacetone and Ethylene Glycol: Synthesis, Characterization, and Nanoparticle Formulation, Biomacromolecules. 7 (2006) 3245-3251.

[5] J. Simon, J.V. Olsson, H. Kim, I.F. Tenney, R.M. Waymouth, Semicrystalline Dihydroxyacetone Copolymers Derived from Glycerol, Macromolecules. 45 (2012) 9275-9281. doi:10.1021/ma302311h.

[6] L.-S. Wang, S.-X. Cheng, R.-X. Zhuo, Syntheses and properties of novel copolymers of polycaprolactone and aliphatic polycarbonate based on ketal-protected dihydroxyacetone, Polym. Bull. 71 (2014) 47-56. doi:10.1007/s00289-013-1044-7.

[7] L.S. Wang, S.X. Cheng, R.X. Zhuo, Novel biodegradable aliphatic polycarbonate based on ketal protected dihydroxyacetone, Macromol. Rapid Commun. 25 (2004) 959-963. doi:10.1002/marc.200300319.

[8] P.W. Henderson, D.J.M. Kadouch, S.P. Singh, P.N. Zawaneh, J. Weiser, S. Yazdi, et al., A rapidly resorbable hemostatic biomaterial based on dihydroxyacetone, J. Biomed. Mater. Res. 93A (2010) 776-782. doi:10.1002/Jbm.A.32586.

[9] P.N. Zawaneh, S.P. Singh, R.F. Padera, P.W. Henderson, J.A. Spector, D. Putnam, Design of an injectable synthetic and biodegradable surgical biomaterial, P Natl Acad Sci USA. 107 (2010) 11014-11019. doi:10.1073/pnas.0811529107.

[10] J.R. Weiser, P.N. Zawaneh, D. Putnam, Poly(carbonate-ester)s of dihydroxyacetone and lactic acid as potential biomaterials, Biomacromolecules. 12 (2011) 977-986. doi:10.1021/bm101342p.

[11] J.R. Weiser, N.G. Ricapito, A. Yueh, E.L. Weiser, D. Putnam, A mechanistic analysis of the quantitation of alpha-hydroxy ketones by the bicinchoninic acid assay, Anal Biochem. 430 (2012) 116-122. doi:10.1016/j.ab.2012.08.009.

[12] C. Soler, M. Soley, Rapid and delayed effects of epidermal growth factor on gluconeogenesis, Biochem J. 294 (1993) 865-872.

[13] L. Stryer, Biochemistry, 4 ed., W.H. Freeman and Co, New York, 1995.

[14] V. Esmann, Dihydroxyacetone as an intermediate during the metabolism of glycerol and glyceraldehyde in leukocytes from rat, Acta Chem Scand. 22 (1968) 2281-2288.

[15] G. Voskerician, M.S. Shive, R.S. Shawgo, H. von Recum, J.M. Anderson, M.J. Cima, et al., Biocompatibility and biofouling of MEMS drug delivery devices, Biomaterials. 24 (2003) 1959-1967. doi:10.1016/S0142-9612(02)00565-3.

[16] G. Voskerician, R.S. Shawgo, P.A. Hiltner, J.M. Anderson, M.J. Cima, R. Langer, In vivo inflammatory and wound healing effects of gold electrode voltammetry for MEMS micro-reservoir drug delivery device, Ieee Transactions on Biomedical Engineering. 51 (2004) 627-635. doi:10.1109/tbme.2003.821034. 
[17] G. Voskerician, A. Rodriguez, P.H. Gingras, Macroporous condensed poly(tetra fluoroethylene). II. In vivo effect on adhesion formation and tissue integration, J. Biomed. Mater. Res. 82A (2007) 426-435. doi:10.1002/jbm.a.31155.

[18] A. Rodriguez, G. Voskerician, H. Meyerson, S.R. MacEwan, J.M. Anderson, T cell subset distributions following primary and secondary implantation at subcutaneous biomaterial implant sites, J. Biomed. Mater. Res. 85A (2008) 556-565. doi:10.1002/jbm.a.31562.

[19] J.M. Anderson, A. Rodriguez, D.T. Chang, Foreign body reaction to biomaterials, Seminars in Immunology. 20 (2008) 86-100. doi:10.1016/j.smim.2007.11.004.

[20] P.J. Flory, Principles of polymer chemistry, (1953).

[21] O. Martin, L. Averous, Poly(lactic acid): plasticization and properties of biodegradable multiphase systems, Polymer. 42 (2001) 6209-6219.

[22] R.A. Shanks, A. Hodzic, S. Wong, Thermoplastic biopolyester natural fiber composites, J Appl Polym Sci. 91 (2004) 2114-2121. doi:10.1002/app.13289.

[23] J.M. Anderson, M.S. Shive, Biodegradation and biocompatibility of PLA and PLGA microspheres, Advanced Drug Delivery Reviews. 28 (1997) 5-24.

[24] J.R. Weiser, A. Yueh, D. Putnam, Protein release from dihydroxyacetone-based poly(carbonate ester) matrices, Acta Biomater. 9 (2013) 8245-8253.

doi:10.1016/j.actbio.2013.05.020.

[25] M. Helou, J.-M. Brusson, J.-F. Carpentier, S.M. Guillaume, Functionalized polycarbonates from dihydroxyacetone: insights into the immortal ring-opening polymerization of 2,2-dimethoxytrimethylene carbonate, Polym. Chem. 2 (2011) 27892795. doi:10.1039/C1PY00405K.

[26] M.A.H. Shipar, Formation of the Heyns rearrangement products in dihydroxyacetone and glycine Maillard reaction: A computational study, Food Chemistry. 97 (2006) 231243. doi:10.1016/j.foodchem.2005.03.041.

[27] K. Jung, M. Seifert, T. Herrling, J. Fuchs, UV-generated free radicals (FR) in skin: their prevention by sunscreens and their induction by self-tanning agents, Spectrochim Acta a Mol Biomol Spectrosc. 69 (2008) 1423-1428. doi:10.1016/j.saa.2007.09.029.

[28] L. Davis, The structure of dihydroxyacetone in solution, Bioorganic Chemistry. 2 (1973) 197-201. doi:10.1016/0045-2068(73)90023-0.

[29] P.H. Bentley, W. McCrae, An efficient synthesis of symmetrical 1,3-diglycerides, J. Org. Chem. 35 (1970) 2082-2083.

[30] C. Schick, Differential scanning calorimetry (DSC) of semicrystalline polymers, Anal Bioanal Chem. 395 (2009) 1589-1611. doi:10.1007/s00216-009-3169-y.

[31] D.J. Blundell, On the interpretation of multiple melting peaks in poly(ether ether ketone), Polymer. 28 (1987) 2248-2251. doi:10.1016/0032-3861(87)90382-X.

[32] R.A. Kenley, M.O. Lee, T.R. Mahoney, L.M. Sanders, Poly(Lactide-co-Glycolide) Decomposition Kinetics In Vivo and In Vitro, Macromolecules. 20 (1987) 2398-2403.

[33] C.M. Agrawal, K.F. Haas, D.A. Leopold, H.G. Clark, Evaluation of Poly(L-Lactic Acid) as a Material for Intravascular Polymeric Stents, Biomaterials. 13 (1992) 176-182.

[34] A.C. Albertsson, I.K. Varma, Aliphatic polyesters: Synthesis, properties and applications, in: Degradable Aliphatic Polyesters, Springer-Verlag Berlin, Berlin, 2002: pp. 1-40.

[35] L.S. Nair, C.T. Laurencin, Biodegradable polymers as biomaterials, Prog. Polym. Sci. 32 (2007) 762-798. doi:10.1016/j.progpolymsci.2007.05.017. 
[36] Y. Hori, Y. Gonda, Y. Takahashi, T. Hagiwara, Ring-Opening Copolymerization of Butyrolactone with Cyclic Carbonates: New Biodegradable Poly(ester carbonate)s, Macromolecules. 29 (1996) 804-806. doi:10.1021/ma951345i.

[37] A.C. Albertsson, I.K. Varma, Aliphatic polyesters: Synthesis, properties and applications, in: Degradable Aliphatic Polyesters, Springer-Verlag Berlin, Berlin, 2002: pp. $1-40$.

[38] K.E. Uhrich, S.M. Cannizzaro, R.S. Langer, K.M. Shakesheff, Polymeric systems for controlled drug release, Chem. Rev. 99 (1999) 3181-3198. doi:10.1021/cr940351u.

[39] A. Lucke, J. Temar, E. Schnell, G. Schmeer, A. Gopferich, Biodegradable poly(,-lactic acid)-poly(ethylene glycol)-monomethyl ether diblock copolymers: structures and surface properties relevant to their use as biomaterials, Biomaterials. 21 (2000) 2361.

[40] O. Coulembier, P. Degee, J.L. Hedrick, P. Dubois, From controlled ring-opening polymerization to biodegradable aliphatic polyester: Especially poly(beta-malic acid) derivatives, Prog. Polym. Sci. 31 (2006) 723-747.

doi:10.1016/j.progpolymsci.2006.08.004.

[41] W.-K. Lee, J.A. Gardella, Hydrolytic Kinetics of Biodegradable Polyester Monolayers, Langmuir. 16 (2000) 3401-3406. doi:10.1021/la990800r.

[42] C.E. Holy, S.M. Dang, J.E. Davies, M.S. Shoichet, In vitro degradation of a novel poly(lactide-co-glycolide) 75/25 foam, Biomaterials. 20 (1999) 1177-1185.

[43] Y.D. Wang, Y.M. Kim, R. Langer, In vivo degradation characteristics of poly(glycerol sebacate), J Biomed Mater Res. 66A (2003) 192-197.

[44] A.C. Grayson, G. Voskerician, A. Lynn, J.M. Anderson, M.J. Cima, R. Langer, Differential degradation rates in vivo and in vitro of biocompatible poly(lactic acid) and poly(glycolic acid) homo- and co-polymers for a polymeric drug-delivery microchip, J. Biomater. Sci.-Polym. Ed. 15 (2004) 1281-1304.

[45] S.M. Li, M. Vert, Hydrolytic degradation of coral/poly(DL-lactic acid) bioresorbable material, J. Biomater. Sci.-Polym. Ed. 7 (1996) 817-827.

[46] K. Yamaguchi, J.M. Anderson, In Vivo Biocompatibility Studies of Medisorb(R) 65/35 D,L-Lactide Glycolide Copolymer Microspheres, Journal of Controlled Release. 24 (1993) 81-93.

[47] J.M. Anderson, In Vivo Biocompatibility of Implantable Delivery Systems and Biomaterials, Eur J Pharm Biopharm. 40 (1994) 1-8.

[48] Y. OGAWA, H. OKADA, Y. YAMAMOTO, T. SHIMAMOTO, In vivo release profiles of leuprolide acetate from microcapsules prepared with polylactic acids or copoly(lactic/glycolic) acids and in vivo degradation of these polymers, Chemical and Pharmaceutical Bulletin. 36 (1988) 2576-2581. doi:10.1248/cpb.36.2576.

[49] P. Menei, V. Daniel, C. Montero-Menei, M. Brouillard, A. Pouplard-Barthelaix, J.P. Benoit, Biodegradation and brain tissue reaction to poly(D,L-lactide-co-glycolide) microspheres, Biomaterials. 14 (1993) 470-478. doi:10.1016/0142-9612(93)90151-Q.

[50] G.E. Visscher, R.L. Robison, H.V. Maulding, J.W. Fong, J.E. Pearson, G.J. Argentieri, Biodegradation of and tissue reaction to 50:50 poly(DL-lactide-co-glycolide) microcapsules, J. Biomed. Mater. Res. 19 (1985) 349-365.

[51] B. Ronneberger, T. Kissel, J.M. Anderson, Biocompatibility of ABA triblock copolymer microparticles consisting of poly(l-lactic-co-glycolic-acid) A-blocks attached to central poly(oxyethylene) B-blocks in rats after intramuscular injection, European Journal of Pharmaceutics and Biopharmaceutics. 43 (1997) 19-28. doi:10.1016/S0939- 
6411(96)00006-9.

[52] B. Ronneberger, W.J. Kao, J.M. Anderson, T. Kissel, In vivo biocompatibility study of ABA triblock copolymers consisting of poly(L-lactic-co-glycolic acid) A blocks attached to central poly(oxyethylene) B blocks, J Biomed Mater Res. 30 (1996) 31-40. doi:10.1002/(SICI)1097-4636(199601)30:1<31::AID-JBM5>3.0.CO;2-S. 


\section{SCHEMATIC, FIGURE AND TABLE LEGENDS}

Scheme 1: Synthetic route to the DHA-based polyesters: recrystallized dihydroxyacetone (i), hexanedioyl dichloride (ii), octanedioyl dichloride (iii), and decanedioyl dichloride (iv), (a) $\mathrm{N}_{2}$ (g), chloroform and pyridine $/ \mathrm{RT}=25^{\circ} \mathrm{C}$, poly(1,3-2-oxo-propylene 1,6-hexanedicarboxylate) (v), poly(1,3-2-oxo-propylene 1,6-octanedicarboxylate) (vi) , and poly(1,3-2-oxo-propylene 1,6octanedicarboxylate) (vii).

Figure 1: $\mathrm{M}_{\mathrm{w}}$ vs. reaction time for poly(DHA-co-adipic acid), poly(DHA-co-suberic acid) and poly(DHA-co-sebacic acid).

Figure 2: Polydispersity vs. reaction time for poly(DHA-co-adipic acid), poly(DHA-co-suberic acid) and poly(DHA-co-sebacic acid).

Figure 3: ${ }^{1} \mathrm{H}$ NMR and ${ }^{13} \mathrm{C}$ NMR spectra of poly(DHA-co-sebacic acid), representative of the copolymers reported herein.

Figure 4: FTIR spectra of poly(DHA-co-sebacic acid), representative of the copolymers reported herein.

Figure 5: In vitro degradation of poly(DHA-co-sebacic acid) (29.6k), $\left(\mathrm{M}_{\mathrm{w}}\right)$ as a function of time at $\mathrm{pHs} 3.0,5.0$ and 7.4 , representative of the copolymers reported herein. Mean values $\pm \mathrm{SD}, \mathrm{n}=3$ Figure 6: In vivo degradation of poly(DHA-co-suberic acid) (9.8k) for both molecular weight and mass loss as a function of time. Mean values $\pm \mathrm{SD}, \mathrm{n}=4$.

Figure 7: Histology of poly(DHA-co-suberic acid)(9.8k), poly(DHA-co-suberic acid)(16.5k), poly(DHA-co-sebacic acid)(18.7k), and poly(DHA-co-sebacic acid)(29.6k) implanted subcutaneously in rats for 16 week. Pictures on the left and right are stained with Hematoxylin and Eosin (H\&E) and Masson's Trichrome (Tri), respectively. Scale bar indicates $500 \mu \mathrm{m}$.

Figure 8: The wound healing response of poly(DHA-co-suberic acid) (9.8k), poly(DHA-cosuberic acid) (16.5k), poly(DHA-co-sebacic acid) (18.7k), and poly(DHA-co-sebacic acid) (29.6k) after 16 weeks in vivo. Scoring system: $0=$ None,$+1=$ Minimal, $+2=$ Mild,$+3=$ Moderate and $+4=$ Extensive. Mean values $\pm \mathrm{SD}, \mathrm{n}=4$ 
Table 1: Maximum $M_{w}$ and $M_{n}$ achieved for each polymer

Table 2: Thermal transition characteristics of DHA-diacid copolymers.

Table 3: Thermal degradation characteristics of DHA-diacid copolymers.

Table 4: In vitro half-lives and degradation rate through 1 half-life.

Table 5: In vivo half-lives and degradation rate through 2 half-lives. 
Schematic 1 Korley et al.

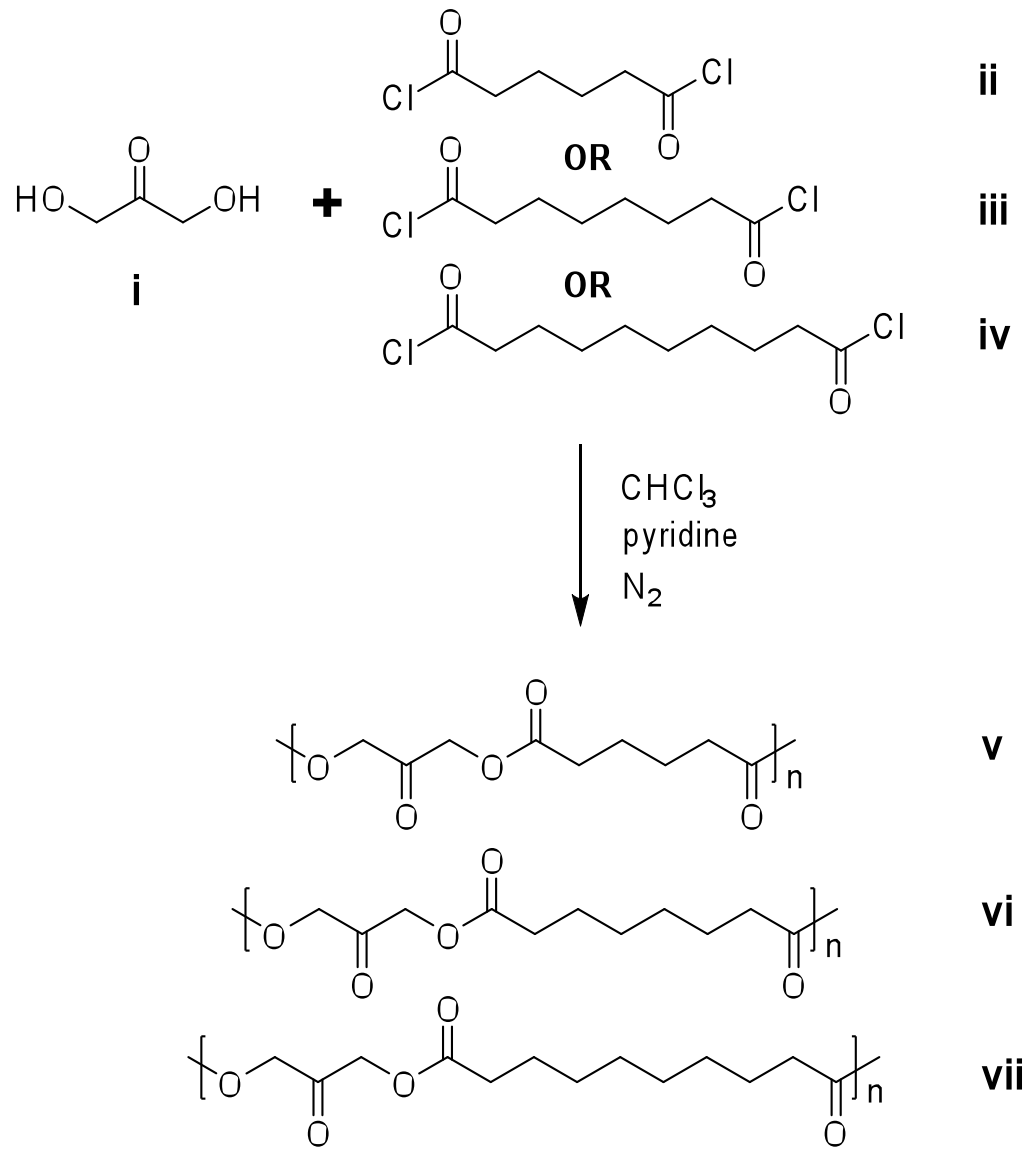


Figure 1 Korley et al

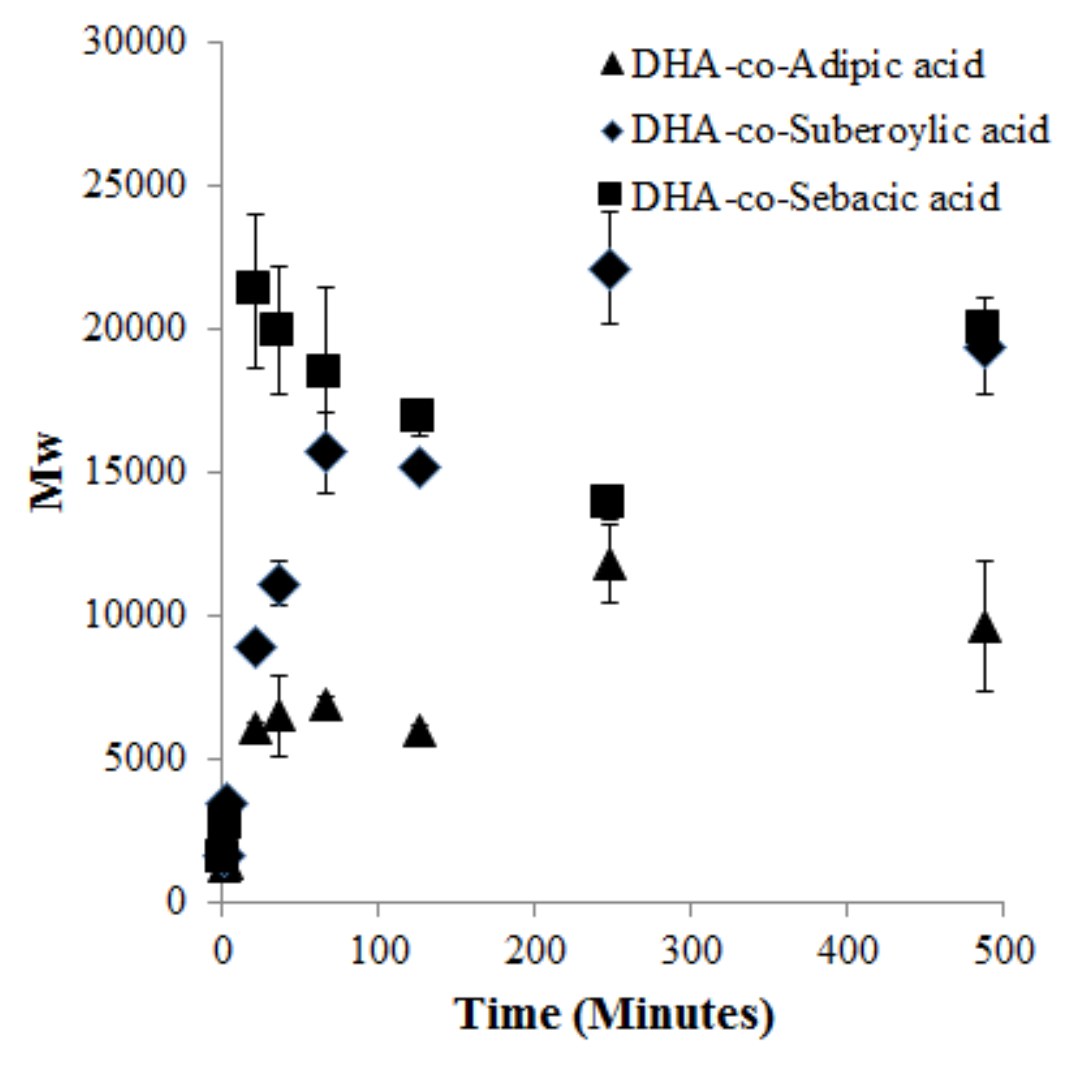


Figure 2 Korley et al

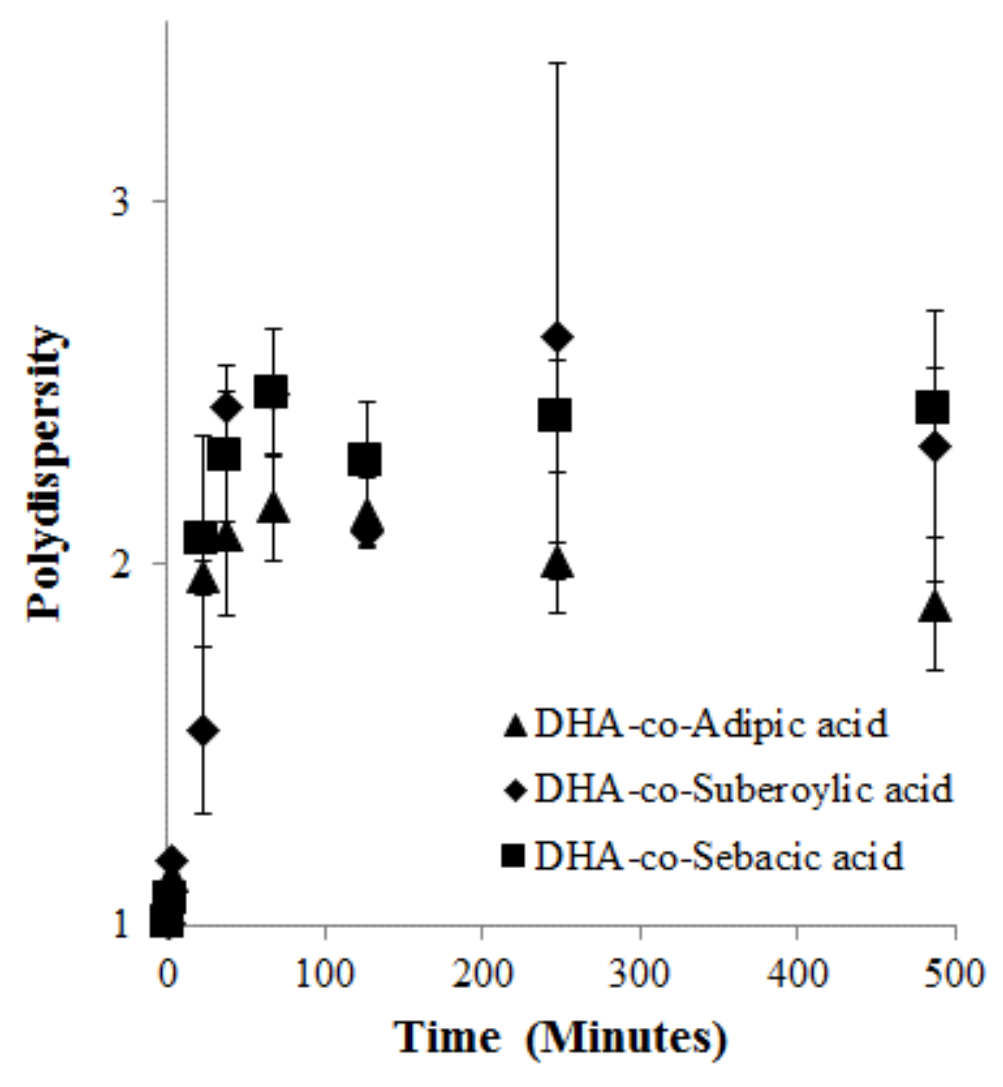


Figure 3 Korley et al

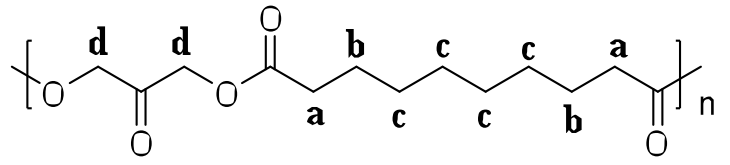
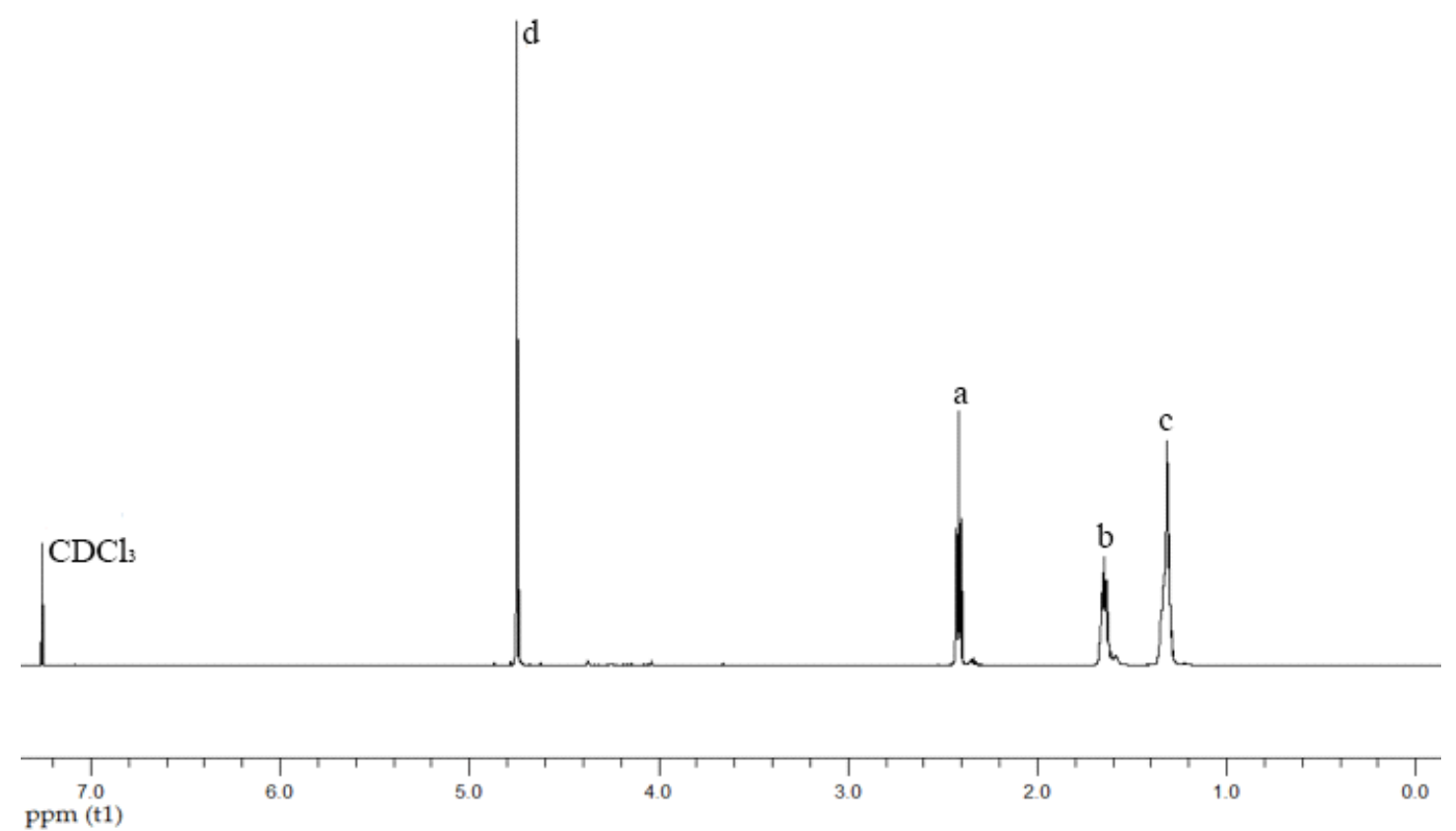

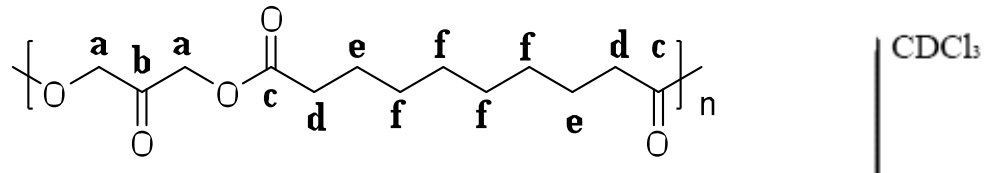
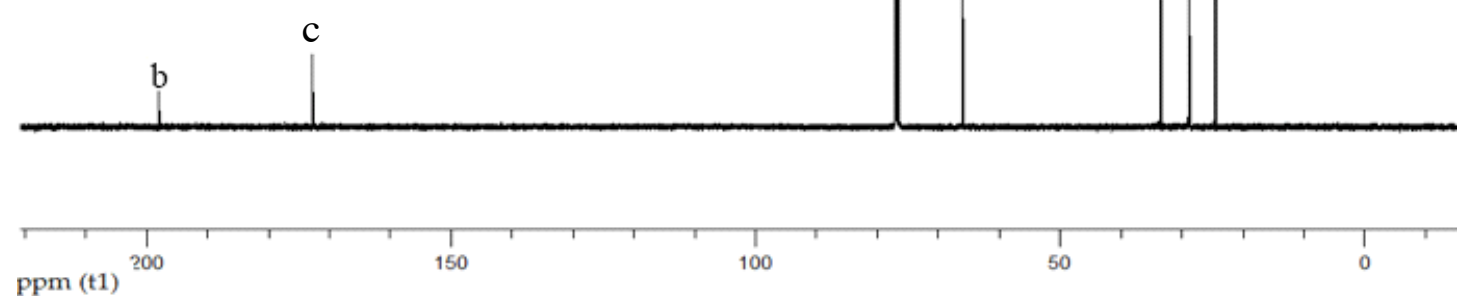
Figure 4 Korley et al

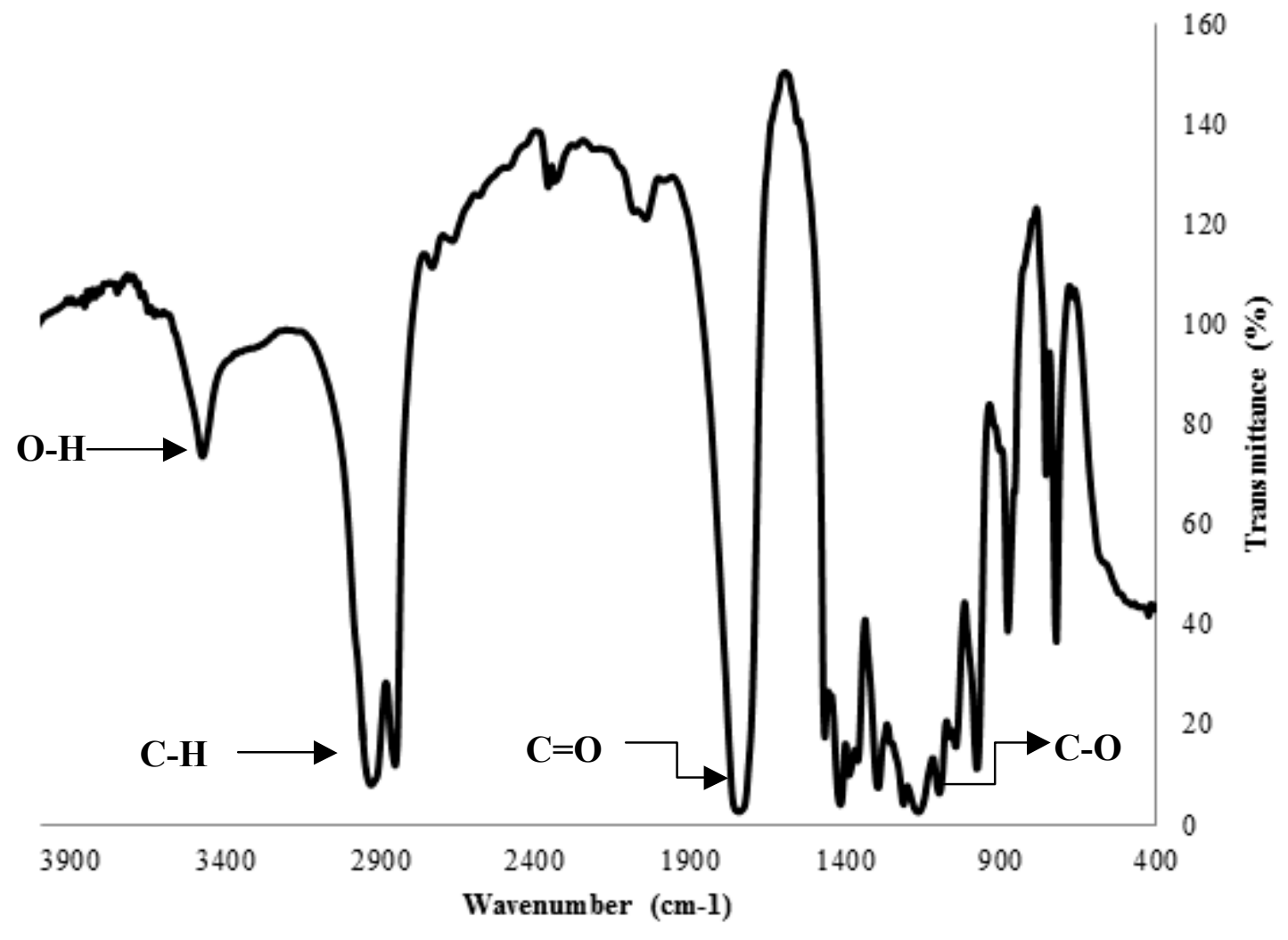


Figure 5 Korley et al

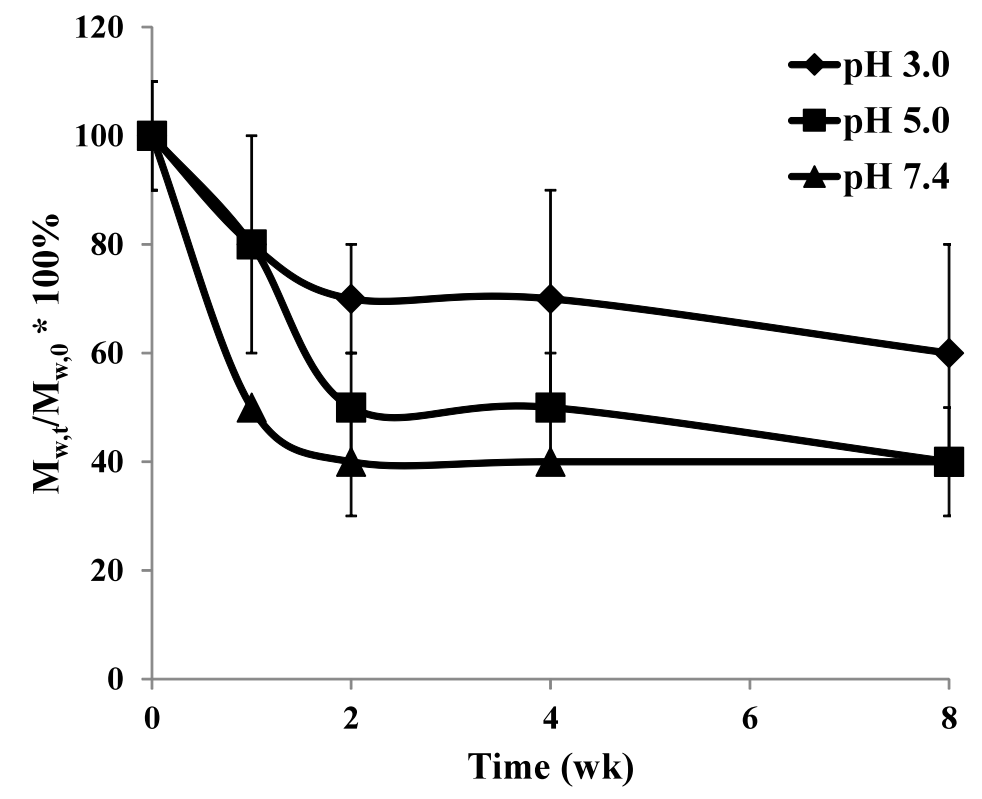


Figure 6 Korley et al

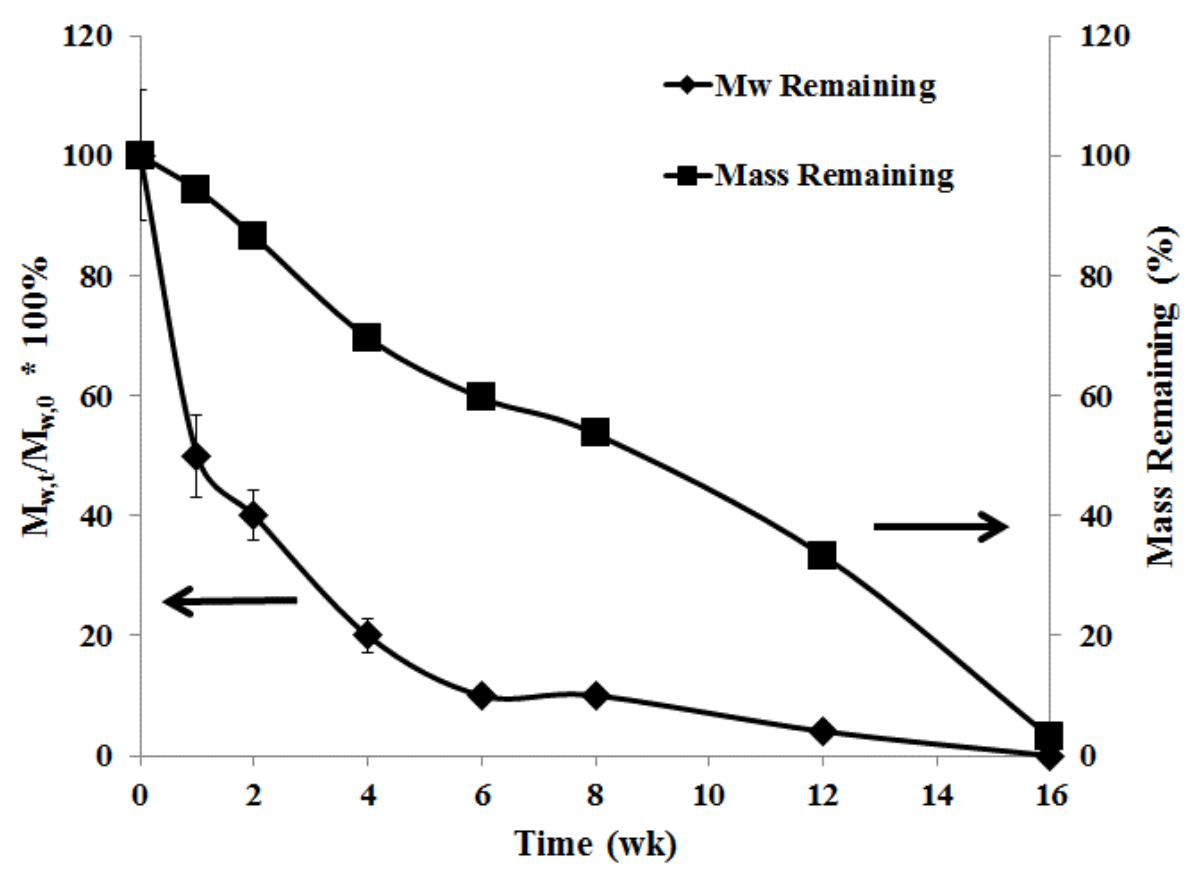


Figure 7 Korley et al

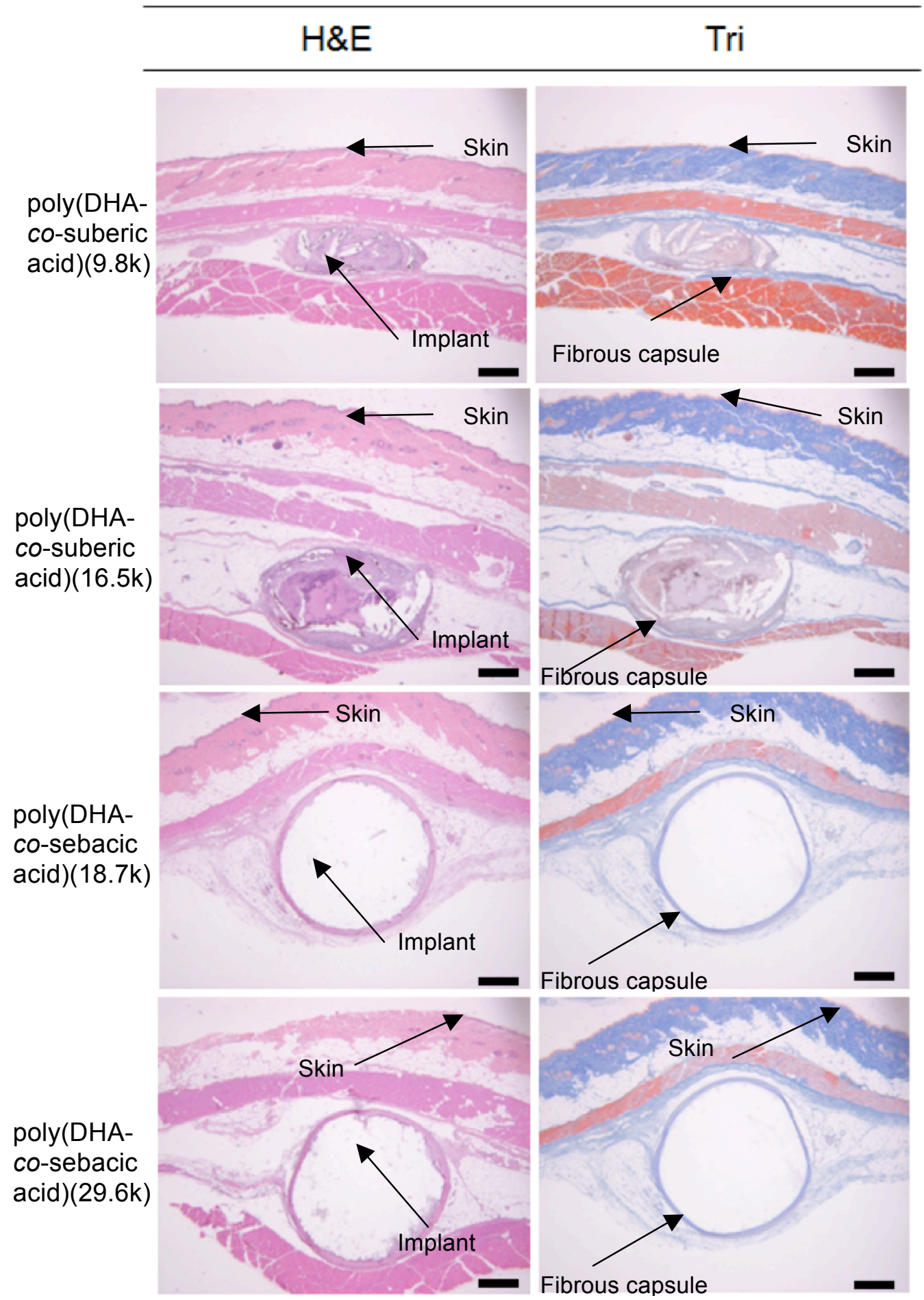


Figure 8 Korley et al

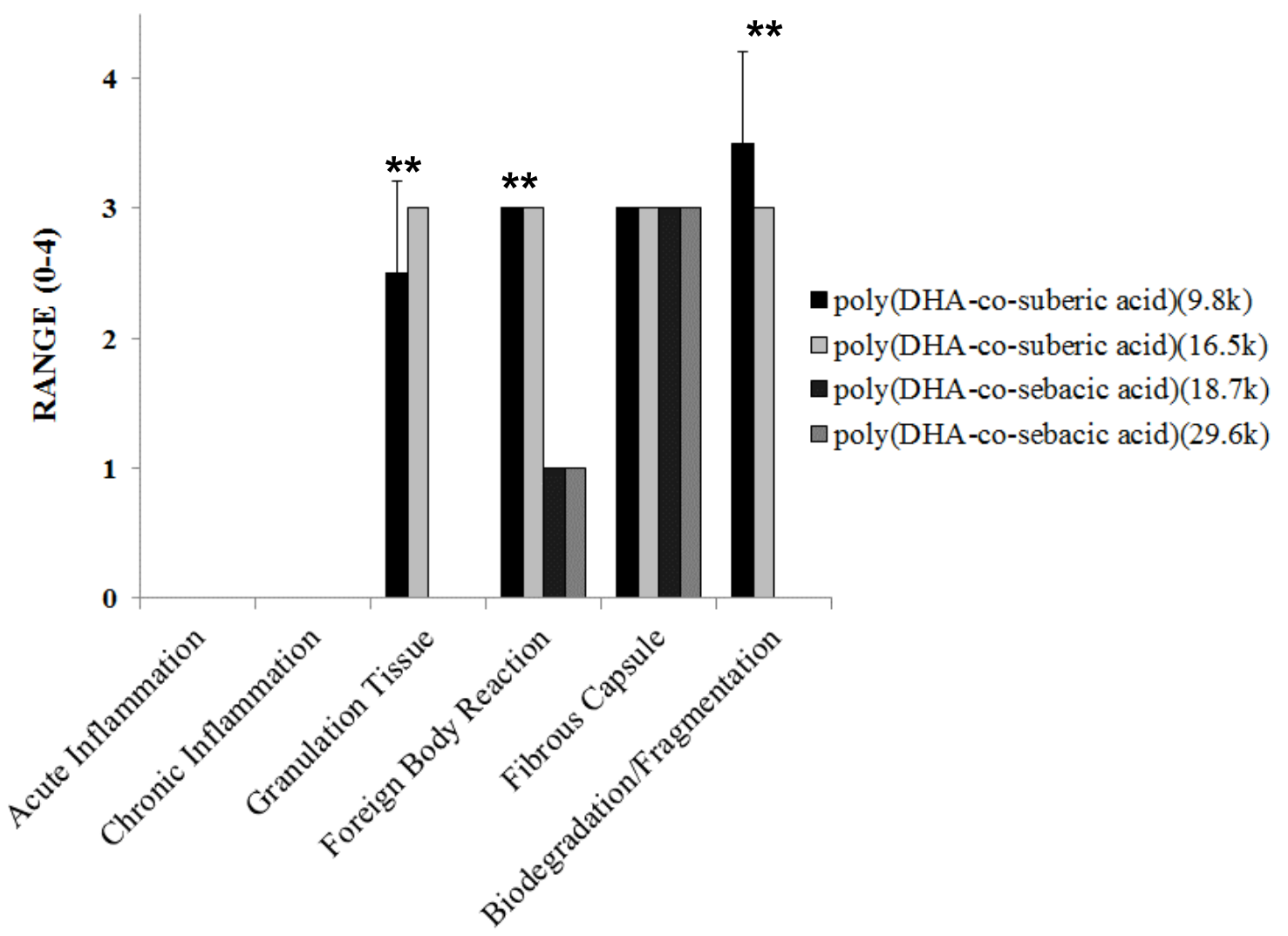


Table 1 Korley et al

\begin{tabular}{cccc}
\hline Chemical Name & $\mathbf{M}_{\mathbf{w}}$ & $\mathbf{M}_{\mathbf{n}}$ & Common Name \\
\hline $\begin{array}{c}\text { Poly(1,3-2-oxo-propylene 1,6- } \\
\text { hexanedicarboxylate) }\end{array}$ & $4.9 \mathrm{~K}$ & $2.2 \mathrm{~K}$ & $\begin{array}{c}\text { Poly(DHA-co-adipic acid) } \\
(4.9 \mathrm{k})\end{array}$ \\
$\begin{array}{c}\text { Poly(1,3-2-oxo-propylene 1,6- } \\
\text { octanedicarboxylate) }\end{array}$ & $9.8 \mathrm{~K}$ & $4.2 \mathrm{~K}$ & $\begin{array}{c}\text { Poly(DHA-co-suberic } \\
\text { acid) }(9.8 \mathrm{k})\end{array}$ \\
$\begin{array}{c}\text { Poly(1,3-2-oxo-propylene 1,6- } \\
\text { octanedicarboxylate) }\end{array}$ & $16.5 \mathrm{~K}$ & $7.2 \mathrm{~K}$ & $\begin{array}{c}\text { Poly(DHA-co-suberic } \\
\text { acid) }(16.5 \mathrm{k})\end{array}$ \\
$\begin{array}{c}\text { Poly(1,3-2-oxo-propylene 1,6- } \\
\text { decanedicarboxylate) }\end{array}$ & $18.7 \mathrm{~K}$ & $7.6 \mathrm{~K}$ & $\begin{array}{c}\text { Poly(DHA-co-sebacic } \\
\text { acid) }(18.7 \mathrm{k})\end{array}$ \\
$\begin{array}{c}\text { Poly(1,3-2-oxo-propylene 1,6- } \\
\text { decanedicarboxylate) }\end{array}$ & $29.6 \mathrm{~K}$ & $11.5 \mathrm{~K}$ & $\begin{array}{c}\text { Poly(DHA-co-sebacic } \\
\text { acid) }(29.6 \mathrm{k})\end{array}$ \\
\hline
\end{tabular}


Table 2 Korley et al

\begin{tabular}{ccccc}
\hline Sample & $\mathbf{T}_{\mathbf{m} 2}\left(\mathbf{C}^{\circ}\right)$ & $\mathbf{\Delta H}_{\mathbf{m}}(\mathbf{m W} / \mathbf{g})$ & $\mathbf{T}_{\mathbf{c} 2}\left(\mathbf{C}^{\circ}\right)$ & $\mathbf{s}_{\mathbf{c}}(\mathbf{m W} \mathbf{g})$ \\
\hline $\begin{array}{c}\text { Poly(DHA-co-adipic acid) } \\
(4.9 \mathrm{k})\end{array}$ & 112 & 72 & 77 & 76 \\
$\begin{array}{c}\text { Poly(DHA-co-suberic acid) } \\
\quad(9.8 \mathrm{k})\end{array}$ & 101 & 61 & 71 & 71 \\
$\begin{array}{c}\text { Poly(DHA-co-suberic acid) } \\
(16.5 \mathrm{k})\end{array}$ & 103 & 69 & 75 & 66 \\
$\begin{array}{c}\text { Poly(DHA-co-sebacic acid) } \\
\quad(18.7 \mathrm{k})\end{array}$ & 119 & 67 & 92 & 74 \\
$\begin{array}{c}\text { Poly(DHA-co-sebacic acid) } \\
(29.6 \mathrm{k})\end{array}$ & 119 & 99 & 87 & 83 \\
\hline
\end{tabular}


Table 3 Korley et al

\begin{tabular}{cc}
\hline Sample & $\mathbf{0 . 5 \%} \mathbf{W t}$ loss $\left({ }^{\circ} \mathbf{C}\right)$ \\
\hline DHA & 73 \\
Adipic acid & 48 \\
Suberic acid & 50 \\
Sebacic acid & 67 \\
Poly(DHA-co-adipic acid) (4.9k) & 139 \\
Poly(DHA-co-suberic acid) (9.8k) & 152 \\
Poly(DHA-co-suberic acid) (16.5k) & 178 \\
Poly(DHA-co-sebacic acid) (18.7k) & 143 \\
Poly(DHA-co-sebacic acid) (29.6k) & 191 \\
\hline
\end{tabular}




\section{Table 4 Korley et al}

\begin{tabular}{|c|c|c|c|c|c|c|c|}
\hline Polymer & pH & $\mathrm{k}_{1}\left(\mathrm{wk}^{-1}\right)$ & $k_{2}\left(w^{-1}\right)$ & $t_{1 / 2}(w k)$ & $t_{2(1 / 2)}(w k)$ & $\mathbf{t}_{1 / 2}$ (day) & $t_{2(1 / 2)}$ (day) \\
\hline \multirow[t]{3}{*}{$\begin{array}{l}\text { Poly(DHA-co-suberic } \\
\text { acid) }(9.8 \mathrm{k})\end{array}$} & 3.0 & 0.147 & N/A & 4.7 & N/A & 33 & N/A \\
\hline & 5.0 & 0.113 & N/A & 6.1 & N/A & 43 & N/A \\
\hline & 7.4 & 0.414 & 0.0062 & 1.7 & 111.8 & 12 & 82 \\
\hline \multirow{4}{*}{$\begin{array}{l}\text { Poly(DHA-co-suberic } \\
\text { acid) }(16.5 \mathrm{k})\end{array}$} & & & & & & & \\
\hline & 3.0 & 0.127 & N/A & 5.5 & N/A & 38 & N/A \\
\hline & 5.0 & 0.093 & N/A & 7.4 & N/A & 52 & N/A \\
\hline & 7.4 & 0.710 & 0.0372 & 1.0 & 18.6 & 7 & 48 \\
\hline \multirow{4}{*}{$\begin{array}{l}\text { Poly(DHA-co-sebacic } \\
\text { acid) }(18.7 \mathrm{k})\end{array}$} & & & & & & & \\
\hline & 3.0 & 0.056 & N/A & 12.5 & N/A & 87 & N/A \\
\hline & 5.0 & 0.068 & N/A & 10.3 & N/A & 72 & N/A \\
\hline & 7.4 & 0.110 & N/A & 6.3 & N/A & 44 & N/A \\
\hline \multirow{4}{*}{$\begin{array}{l}\text { Poly(DHA-co-sebacic } \\
\text { acid) }(29.6 \mathrm{k})\end{array}$} & & & & & & & \\
\hline & 3.0 & 0.061 & N/A & 11.4 & $\mathrm{~N} / \mathrm{A}$ & 80 & N/A \\
\hline & 5.0 & 0.118 & N/A & 5.9 & $\mathrm{~N} / \mathrm{A}$ & 41 & N/A \\
\hline & 7.4 & 0.453 & 0.0226 & 1.5 & 30.7 & 11 & 75 \\
\hline
\end{tabular}


Table 5 Korley et al

\begin{tabular}{cccc}
\hline Polymer & $\mathbf{k}\left(\mathbf{w k}^{-\mathbf{1}}\right)$ & $\mathbf{t}_{\mathbf{1} / \mathbf{2}}$ (wk) & $\mathbf{t}_{\mathbf{1} / \mathbf{2}}$ (day) \\
\hline Poly(DHA-co-suberic acid) $(9.8 \mathrm{k})$ & $0.330 \pm 0.003$ & 2.2 & 15 \\
Poly(DHA-co-suberic acid) (16.5k) & $0.307 \pm 0.009$ & 2.3 & 16 \\
Poly(DHA-co-sebacic acid) (18.7k) & $0.230 \pm 0.011$ & 3.0 & 21 \\
Poly(DHA-co-sebacic acid) (29.6k) & $0.162 \pm 0.004$ & 4.3 & 30 \\
\hline
\end{tabular}

